\title{
Flavour anomalies from a split dark sector
}

\author{
Luc Darmé, ${ }^{a}$ Marco Fedele, ${ }^{b}$ Kamila Kowalska ${ }^{c}$ and Enrico Maria Sessolo ${ }^{c}$ \\ ${ }^{a}$ INFN - Laboratori Nazionali di Frascati, \\ C.P. 13, 100044 Frascati, Italy \\ ${ }^{b}$ Departament de Física Quàntica i Astrofísica, Institut de Ciències del Cosmos (ICCUB), \\ Universitat de Barcelona, Martí i Franquès 1, E-08028 Barcelona, Spain \\ ${ }^{c}$ National Centre for Nuclear Research, \\ ul. Pasteura 7, 02-093 Warsaw, Poland \\ E-mail: luc.darme@lnf.infn.it, marco.fedele@icc.ub.edu, \\ kamila.kowalska@ncbj.gov.pl, enrico.sessolo@ncbj.gov.pl
}

Abstract: We investigate solutions to the flavour anomalies in $B$ decays based on loop diagrams of a "split" dark sector characterised by the simultaneous presence of heavy particles at the $\mathrm{TeV}$ scale and light particles around and below the $B$-meson mass scale. We show that viable parameter space exists for solutions based on penguin diagrams with a vector mediator, while minimal constructions relying on box diagrams are in strong tension with the constraints from the LHC, LEP, and the anomalous magnetic moment of the muon. In particular, we highlight a regime where the mediator lies close to the $B$-meson mass, naturally realising a resonance structure and a $q^{2}$-dependent effective coupling. We perform a full fit to the relevant flavour observables and analyse the constraints from intensity frontier experiments. Besides new measurements of the anomalous magnetic moment of the muon, we find that decays of the $B$ meson, $B_{s}$-mixing, missing energy searches at Belle-II, and LHC searches for top/bottom partners can robustly test these scenarios in the near future.

KEYwORDS: Beyond Standard Model, Heavy Quark Physics

ARXIV EPRINT: 2002.11150 


\section{Contents}

1 Introduction 1

2 Effective one-loop Wilson coefficients from split dark sector models 3

2.1 Box diagrams 3

2.2 Penguin diagrams 5

3 Constraints on the model $\quad 9$

$\begin{array}{llr}3.1 & \text { Flavour constraints } & 9\end{array}$

$\begin{array}{ll}3.2 & \text { Precision physics constraints } \\ \end{array}$

$\begin{array}{lll}4 & \text { Fitting procedure and results } & 15\end{array}$

5 Conclusions $\quad 21$

A Invisible decay limits $\quad 22$

\section{Introduction}

Several flavour anomalies have been observed in the last few years in various $B$-meson decay measurements by different experimental collaborations. Some of the anomalous measurements involve semileptonic $b \rightarrow s$ transitions and include: (1) the suppression with respect to the Standard Model (SM) expectation of the ratios $R_{K}$ and $R_{K^{*}}$ - the branching ratios of the $B$-meson decay into a $K$ or $K^{*}$ meson and muons, over the decay to the same kaon and electrons - which were observed at LHCb [1-4] and which imply the possible violation of lepton-flavour universality (LFUV); (2) an enhancement in the angular observable $P_{5}^{\prime}$, first measured by the LHCb [5] and Belle collaborations [6], and later observed also by ATLAS and CMS [7, 8]; and (3) a suppression in the branching ratios for the decays $B_{s} \rightarrow \phi \mu^{+} \mu^{-}[9]$ and $B \rightarrow K^{(*)} \mu^{+} \mu^{-}[10,11]$.

Global effective field theory analyses of the $b \rightarrow s$ data have pointed strongly towards New Physics (NP) in the four-fermion operators $\mathcal{O}_{9}^{(\prime) \mu}, \mathcal{O}_{10}^{(\prime) \mu}[12-31]$. Different combinations of the corresponding Wilson coefficients seem to be equally favoured, as long as $C_{9}^{\mu}$ remains significantly below zero. For instance, in a single operator scenario involving only $\mathcal{O}_{9}^{\mu}$, a solution consistent with the full set of $b \rightarrow s$ measurements requires approximately $C_{9}^{\mu} \in[-1.2,-0.6]$ at the $2 \sigma$ level.

While the most natural assumption is that heavy states above the scale of electroweak symmetry breaking (EWSB) are responsible for generating these operators, alternative possibilities have emerged in the literature [18,32-36], that these NP effects are in fact due to the presence of light degrees of freedom around or below the typical scale proved by the experiment. 
The solutions based on a light dark sector can be divided roughly into two categories, depending on whether the masses involved lie above or below the characteristic window for LFUV observables, which is commonly identified as ranging roughly from the dimuon threshold to $\sqrt{6}-\sqrt{8} \mathrm{GeV}$. The first category involves a new light vector with mass $m_{V} \gtrsim 2 \mathrm{GeV}$, coupled to the $b-s$ and the muon currents, interfering negatively with the SM amplitude $[18,33]$. The light particle can contribute strongly to the physical observables thanks to the proximity of a resonance to the experimental bins [33] and NP effects can be parametrised in this case by Wilson coefficients with an explicit $q^{2}$ dependence. Interestingly, the corresponding resonance in the dimuon spectrum from $B$-meson decay could be hidden due to the sizeable hadronic uncertainty and the presence of the $J / \Psi$ resonance in the same region $[33,37]$.

A second class of models, featuring instead the exchange of light particles below the dimuon threshold, have been considered in refs. [18, 32, 34, 35]. These scenarios are strongly constrained by a number of observations. On the one hand, a light scalar particle with effective couplings to the $s$ and $b$ quarks and leptons yields a positive contribution to the decay rate. One thus requires a sizeable coupling to electrons, a scenario that is in most cases [34] in tension with the measurement of $B \rightarrow K^{(*)} e^{+} e^{-}$processes in agreement with the SM [38]. On the other hand, a light vector boson with effective couplings to the $s$ and $b$ quark and muons can interfere negatively with the SM process, but is strongly constrained by the measurement of the anomalous magnetic moment $(g-2)_{\mu}$. This in turn implies a sizeable coupling to the $b$ and $s$ quarks, leading to strong bounds from $B_{s}$ mixing. Finally, in ref. [35] it was pointed out that a resonance coupling to electrons can actually be used to reproduce the low- $q^{2}$ bin of $R_{K^{*}}$. A vector boson very close - but below - the dimuon threshold, where $\mathcal{B R}\left(B \rightarrow K^{(*)} V\right)$ can be as small as $1 \times 10^{-7}$, can explain the anomaly and escape the limits from ref. [38].

A common trait of the constructions mentioned above is the presence of an effective off-diagonal coupling $g_{b s}$ to the quark current. Since the quarks carry SU $(3)_{c}$ charge, $g_{b s}$ must arise from particles with colour integrated out in the UV theory, which must be heavy to avoid exclusion by the strong limits from the LHC. This also means that in realistic scenarios $g_{b s}$ will be suppressed by either loop effects, a small tree-level mixing angle between the SM quarks and heavy vector-like (VL) particles, or a combination of both.

In this paper we perform a detailed exploration of the first of these possibilities, i.e, the UV-complete model gives rise to the effective $b-s$ coupling of the light particle via loop effects. We will show that, under these assumptions, in order to generate a $g_{b s}$ large enough to fit the flavour anomalies and, at the same time, maintain reasonable agreement with perturbativity of the couplings, several phenomenological challenges must be faced. In the specific of the cases mentioned above, we will show that solutions with $m_{V} \gtrsim$ $2 \mathrm{GeV}$ can be made viable with appropriate UV completions, even though they are subject to a combination of increasingly tightening bounds that include, in the UV, LHC direct constraints on hadronic and leptonic new states and Drell-Yan dimuon constraints from the $Z$ lineshape, and in the IR, an appropriate width- and bin-dependent treatment of the bounds from $\mathcal{B R}(B \rightarrow K+$ invisible $)$ and $\mathcal{B R}(B \rightarrow K \mu \mu)$. Conversely, the second class of solutions, characterised by $m_{V}$ under the dimuon threshold, is less appealing, as is strongly 


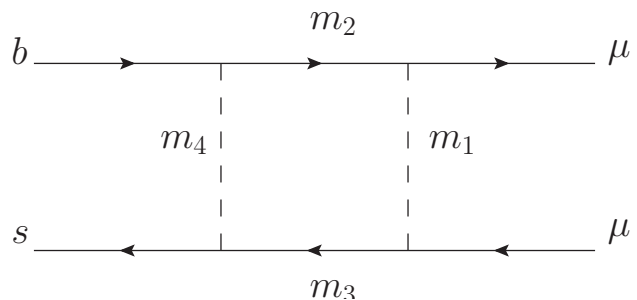

(a)

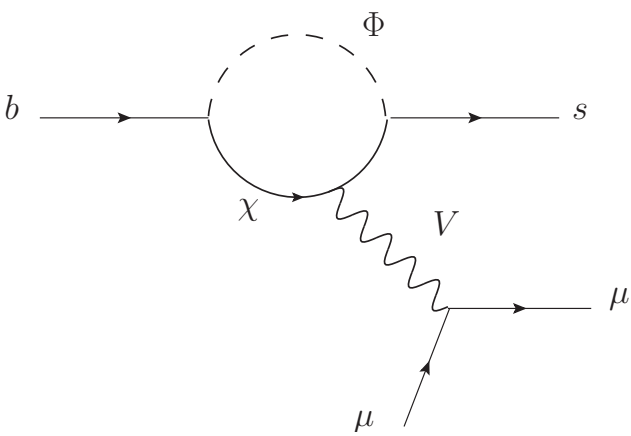

(b)

Figure 1. (a) Example box loop mediating the $B$-meson decay. (b) Example penguin loop mediating the $B$-meson decay.

constrained by a combination of bounds from $\mathcal{B R}(B \rightarrow K+$ invisible $)$, neutrino trident production, intensity frontier limits on kinetic mixing, and $\mathcal{B R}\left(B_{s} \rightarrow \mu \mu\right)$.

More in general, we perform in this work a detailed Monte Carlo scan of the broad $m_{V}$ range and the loop-induced couplings. We identify and characterise the specific properties of the viable models and provide an indication of possible strategies for a timely detection of the associated NP states.

The paper is organised as follows. In section 2 we recall expressions for the loopgenerated Wilson coefficients from box and penguin diagrams. We provide the quantum numbers of the particles entering the loops and estimate the characteristic size of the couplings required to fit the flavor anomalies. In section 3 we enlist the complete set of constraints we apply to the models. Section 4 is dedicated to the main results, with a description of the fitting procedure and discussion of the allowed parameter space. We present our conclusions in section 5. Appendix A is dedicated to the detailed treatment of the recasting procedure for $B \rightarrow K+$ inv. limits.

\section{Effective one-loop Wilson coefficients from split dark sector models}

Our goal is to investigate to what extent the $b-s$ flavour anomalies can be explained by generic loop effects involving light new particles (in association with a heavy sector above the EWSB scale). Possible constructions for the Wilson coefficients of the effective Hamiltonian descending from loops of $\mathrm{TeV}$-scale new particles have been investigated, e.g., in refs. [39-46]. They generally involve either box diagrams of scalar and fermionic states like in figure 1(a), or penguin diagrams like the ones represented in figure 1(b).

\subsection{Box diagrams}

If direct Yukawa couplings between the quarks $b, s$, the muons, and a NP sector composed of fermions $\psi_{i}$ and scalars $\phi_{j}$ are allowed by the gauge quantum numbers, the most generic 
Lagrangian is given by

$$
\begin{aligned}
\mathcal{L} \supset & \bar{\psi}_{i}\left(Y_{L, i j}^{(b)} P_{L} b+Y_{L, i j}^{(s)} P_{L} s+Y_{L, i j}^{(\mu)} P_{L} \mu\right) \phi_{j} \\
& +\bar{\psi}_{k}\left(Y_{R, k l}^{(b)} P_{R} b+Y_{R, k l}^{(s)} P_{R} s+Y_{R, k l}^{(\mu)} P_{R} \mu\right) \phi_{l}+\text { H.c. },
\end{aligned}
$$

where a sum over repeated indices is implied. It was shown, e.g., in refs. [39, 40, 46, 47] that box diagrams like the one depicted in figure 1(a) can then contribute to the $B \rightarrow K^{(*)} \mu^{+} \mu^{-}$ decay and one can construct out of eq. (2.1) Wilson coefficients $C_{9}^{(\prime) \mu}, C_{10}^{(\prime) \mu}$ of the right order of magnitude to fit the anomalies. On the other hand, these box-like constructions do not generally involve light particles or very split spectra, as they would incur strong tension with several existing bounds.

Since at least one of the fermion or scalar fields in the box must necessarily carry the colour charge, the bounds from LHC searches for colour production with $b$-tagged jets will contribute to push this state above the $1-1.2 \mathrm{TeV}$ scale [48-51]. At the same time there exist strong bounds from the measurement of $B_{s}$ mixing [52] that either limit the available size of the Yukawa couplings or push one of the new states coupled to $b, s$ to a very large scale. The strength of the $B_{s}$-mixing bound is very model-dependent and, if the particle content of the model at hand is large enough, cancellations between different diagrams can be engineered to evade the limit. Nevertheless, we can easily obtain an estimate of the $B_{s}$-mixing bound in minimal cases, when the particle content is just about right to fit the flavour anomalies via a single box diagram similar to figure 1(a). Assuming that the colour charge is carried by particle 4 in the figure ${ }^{1}$ we get, following, e.g., ref. [40], $\left|Y_{L, 24}^{(b)} Y_{L, 34}^{(s) *}\right| \lesssim 0.09$ at the $2 \sigma$ level.

If particle 4 carries colour, the other states can in principle be lighter: $m_{1} \approx m_{2} \approx$ $m_{3} \lesssim m_{4} \approx \mathcal{O}(\mathrm{TeV})$. The Wilson coefficient $C_{9}^{\mu}$ is then approximately calculated as (see, e.g., refs. $[40,46])$

$$
C_{9}^{\mu} \approx-\frac{Y_{L}^{(s) *} Y_{L}^{(b)}\left|Y_{L, R}^{(\mu)}\right|^{2}}{\left(m_{4} / \mathrm{TeV}\right)^{2}} F(x, y, z),
$$

where we have suppressed the subscript indices in the Yukawa couplings to lighten the notation, we define $x=\left(m_{1} / m_{4}\right)^{2}, y=\left(m_{2} / m_{4}\right)^{2}, z=\left(m_{3} / m_{4}\right)^{2}$, and $F$ is a loop function,

$$
F(x, y, z)=\frac{x^{2} \ln x}{(x-1)(x-y)(x-z)}+\frac{y^{2} \ln y}{(y-1)(y-x)(y-z)}+\frac{z^{2} \ln z}{(z-1)(z-x)(z-y)},
$$

which equals approximately 1 when $m_{1} \approx m_{2} \approx m_{3} \approx \mathcal{O}(100 \mathrm{GeV})$. As was shown in, e.g., refs. [39, 40, 46, 47], it follows from eq. (2.2) that $C_{9}^{\mu} \lesssim-0.6$ requires Yukawa couplings $\left|Y_{L, R}^{(\mu)}\right| \gtrsim 3$ for $m_{1} \approx m_{2} \approx m_{3} \approx \mathcal{O}(100 \mathrm{~s} \mathrm{GeV})$, a condition that does not guarantee the validity of perturbation theory up to scales much larger than EWSB.

\footnotetext{
${ }^{1}$ The opposite choice would imply that particles 1,2 , and 3 carry colour, which in turn would lead to most states in the loop being either at the $\mathrm{TeV}$ scale or, in fact, SM quarks. While the latest possibility is interesting, it would trigger very strong tree-level flavour-violating bounds whose effect are likely to dominate the loop-induced signatures we consider here.
} 
One might wonder at this point if an eventual light sector in the theory can provide a more natural fit to the flavour anomalies, perhaps requiring smaller Yukawa couplings to the muons. In fact, one infers from eq. (2.3) that $F$ can receive a logarithmic enhancement of a few units if $x, y$, and $z$ are all at the same time significantly smaller than 1 . But at least one among $m_{1}, m_{2}, m_{3}$ cannot be much smaller than $m_{4}$. There are two reasons for this. First and foremost, multi-lepton searches at the LHC via Drell-Yan production constrain the particles carrying $\mathrm{SU}(2)_{L} \times \mathrm{U}(1)_{Y}$ quantum numbers to masses above the $400-600 \mathrm{GeV}$ range [53-55]. On the other hand, if one were to roughly extrapolate a similar reasoning to $m_{1}, m_{2}, m_{3}$ smaller than the mass of the muon, so that the corresponding particles may possibly result invisible at the LHC, these would still necessarily contribute at one loop to the anomalous magnetic moment of the muon. The measured $2 \sigma$ upper bound, $\delta(g-2)_{\mu} \lesssim 4 \times 10^{-9}[56-58]$ implies that the muon Yukawa coupling cannot be larger than

$$
\left|Y_{i j, L(R)}^{(\mu)}\right| \lesssim 10^{-2}\left(\frac{m_{1,2,3}}{\mathrm{GeV}}\right)
$$

quite independently of the specifics of the model at hand.

A rough comparison of the typical size of eq. (2.2) and eq. (2.4) shows that the latter is too small to fit the flavour anomalies unless $m_{1,2,3}$ lie in the few hundreds of $\mathrm{GeV}$ or above. We conclude that there is arguably no common parameter space for $b \rightarrow s$ anomalies and $(g-2)_{\mu}$ with perturbative Yukawa couplings and a minimal, light NP sector, if the contributions to $B$ decays stem from this class of box diagrams.

\subsection{Penguin diagrams}

It is more promising to look at another type of loop-induced coupling giving rise to the effective operators $\mathcal{O}_{9}^{(\prime)}, \mathcal{O}_{10}^{(\prime)}$ : the penguin-diagram generated interaction. The penguin is constructed out of a loop with new fermion and scalar fields exchanging a vector boson with the leptons. If the vector boson is one of the SM gauge bosons, the contribution to $C_{9}^{(\prime)}, C_{10}^{(\prime)}$ is flavour-universal, and cannot be used to explain the LFUV anomalies. We will therefore be interested in models realising the penguin diagram topology presented in figure 1(b), in which a new light gauge boson $V$ couples to the muons.

The particle that carries the colour charge in the loop might be a scalar multiplet $\Phi$ or a heavy VL fermion $\Psi$. In the former case we close the loop with a light (Dirac) fermion $\chi$, whereas in the latter with a light scalar $\pi$. In order to avoid charging the $b$ and $s$ quarks under the dark gauge group $\mathrm{U}(1)_{D}$ (with gauge coupling $g_{D}$ ), we assume that the dark charge is confined within the loop, i.e., $Q_{\chi(\pi)}=-Q_{\Phi(\Psi)}$. We thus avoid the strong bounds on $V$ from multi-lepton searches at the LHC.

Without much loss of generality we will focus henceforth on the case where the heavy coloured particle is $\Phi$. The case with $\Psi$ does not present very significant differences, barring an order-one suppression of the amplitude that comes from swapping the role of the light and heavy mass in the loop functions. We thus introduce a scalar doublet $\Phi=\left(\phi_{u}, \phi_{d}\right)^{T}$ and a few light fermion singlets $\chi_{i}$, whose multiplicity will be specified case by case. Explicitly, their $\mathrm{SU}(3)_{c} \times \mathrm{SU}(2)_{L} \times \mathrm{U}(1)_{Y} \times \mathrm{U}(1)_{D}$ quantum numbers read

$$
\Phi=\left(\mathbf{3}, \mathbf{2}, 1 / 6, Q_{\Phi}\right) \quad \chi_{i}=\left(\mathbf{1}, \mathbf{1}, 0,-Q_{\Phi}\right) .
$$


Note that with the above charge assignment the mass matrix of the dark fermions is not a priori diagonal, unless the off-diagonal entries are forbidden by a flavour symmetry, or suppressed by some other mechanism. We will assume for simplicity that this is always the case, without entering in the specifics of such constructions.

We confine ourselves to the treatment of left-handed $b-s$ currents, in agreement with the findings of the global fits in the literature. Below the EWSB scale the Lagrangian of the hadronic NP sector reads

$$
\mathcal{L} \supset y_{d}^{i j} \phi_{d}^{*} \bar{\chi}_{i} P_{L} d_{j}+y_{u}^{i j} \phi_{u}^{*} \bar{\chi}_{i} P_{L} u_{j}+\text { H.c. },
$$

where a sum over repeated indices is implied, and the Yukawa couplings are related by $y_{u}^{i j}=y_{d}^{i k}\left(V_{\mathrm{CKM}}^{\dagger}\right)^{k j}$. We further confine ourselves to the basis where the only nonzero Yukawa couplings of the down-like type are those of the second and third generation: $y_{d}^{i k=1,2,3}=\left(0, y_{s}^{i}, y_{b}^{i}\right)^{T}$.

We finally introduce an effective interaction of the new gauge boson to the muons:

$$
\mathcal{L} \supset\left(g_{\mu}^{V} \bar{\mu} \gamma_{\nu} \mu+g_{\mu}^{A} \bar{\mu} \gamma_{\nu} \gamma_{5} \mu\right) V^{\nu} .
$$

The relative size of the $g_{\mu}^{V}$ and $g_{\mu}^{A}$ couplings is governed by the UV model building and so is the eventual size, if nonzero at all, of the coupling of the new gauge boson to neutrinos. This point is of particular relevance when it comes to the constraints on the model from neutrino trident production [59] at CCFR [60] and CHARM-II [61], to which we come back in sections 3 and 4 . We shall see that, while it is desirable to embed the effective model in a framework with forbidden or strongly suppressed couplings to the neutrinos, ${ }^{2}$ we are able to find in our numerical analysis some viable parameter space lying below the bound from CCFR and CHARM-II.

We calculate the penguin diagram contributions to the $B_{s} \rightarrow K^{(*)} \mu^{+} \mu^{-}$decay. Following standard techniques one obtains an amplitude that closely resembles the SM photon penguin case, with a slight modification due to the Breit-Wigner distribution of the massive gauge boson $V$. Estimating the loop diagram is equivalent to integrating out the heavy colour-charged field, which generates the dimensionful Wilson coefficient $\tilde{g}$ of the operator

$$
\mathcal{O}_{6}=\left(\bar{s} \gamma_{\rho} P_{L} b\right) \partial_{\sigma} V^{\rho \sigma}
$$

where $V^{\rho \sigma}=\partial^{\rho} V^{\sigma}-\partial^{\sigma} V^{\rho}[34,35]$. We find

$$
\tilde{g}=-\frac{g_{D} Q_{\Phi}}{16 \pi^{2} m_{\Phi}^{2}} \sum_{i} y_{s}^{i *} y_{b}^{i} \mathcal{F}\left(x_{i}\right),
$$

\footnotetext{
${ }^{2}$ For example, one can construct a Type-I 2-Higgs doublet model where the additional Higgs doublet and extra VL heavy leptons all carry $\mathrm{U}(1)_{D}$ charge: $\Theta:\left(\mathbf{1}, \mathbf{2}, 1 / 2, Q_{\Theta}\right), E:\left(\mathbf{1}, \mathbf{1}, 1, Q_{\Theta}\right)$. Yukawa couplings with the SM left-handed muon doublet $L_{\mu}$, of the type $\lambda \Theta^{\dagger} L_{\mu} E$, generate a left-chiral coupling $g_{\mu}^{L}$ of $V$ to the muons once $\mathrm{U}(1)_{D}$ is broken. The coupling to neutrinos is absent. Typically one gets $g_{\mu}^{L} \approx-g_{D} Q_{\Theta} \lambda v_{\Theta}^{2} / 2 m_{E}^{2}$. More than one family of VL singlet fermions, and an additional complex scalar singlet charged under $\mathrm{U}(1)_{D}$ can be introduced to generate the right-chiral couplings and to push the mass of the scalars with electric charge above the current bound from LEP and LHC searches [62].
} 
where we have defined $x_{i}=m_{\chi_{i}}^{2} / m_{\Phi}^{2}$ and the loop function reads

$$
\mathcal{F}(x)=-\frac{1}{2}-\frac{1}{3} \ln x .
$$

The contribution of $\mathcal{O}_{6}$ to the $B \rightarrow K$ processes mediated by the exchange of a vector boson can be related to "effective" Wilson coefficients $C_{9,10}^{\ell}$ as

$$
C_{9(10)}^{\mu}\left(q^{2}\right)=-\frac{4 \pi \mathcal{N}}{\alpha_{\mathrm{EM}}} \tilde{g} g_{\mu}^{V(A)} \frac{q^{2}}{q^{2}-m_{V}^{2}+i m_{V} \Gamma_{V}},
$$

where $m_{V}$ is the mass of the gauge boson, we define

$$
\mathcal{N}^{-1}=\frac{4 G_{F}}{\sqrt{2}} V_{t b} V_{t s}^{*}
$$

and $\Gamma_{V}$ is the total width of the gauge boson, which reads, when all light decay channels are kinematically open,

$$
\Gamma_{V}=m_{V}\left(\gamma_{V}^{D}+\gamma_{V}^{\mu}\right),
$$

with the dark fermion and SM muon contributions respectively given by

$$
\begin{aligned}
& \gamma_{V}^{D}=\frac{1}{12 \pi}\left[\sum_{i} \sqrt{1-\frac{4 m_{\chi_{i}}^{2}}{m_{V}^{2}}}\left(\frac{2 m_{\chi_{i}}^{2}}{m_{V}^{2}}+1\right) g_{D}^{2} Q_{\chi}^{2}\right], \\
& \gamma_{V}^{\mu}=\frac{1}{12 \pi}\left[\sqrt{1-\frac{4 M_{\mu}^{2}}{m_{V}^{2}}}\left(\frac{2 M_{\mu}^{2}}{m_{V}^{2}}+1\right)\left(g_{\mu}^{V}\right)^{2}+\left(1-\frac{4 M_{\mu}^{2}}{m_{V}^{2}}\right)^{3 / 2}\left(g_{\mu}^{A}\right)^{2}\right] .
\end{aligned}
$$

Note that the Wilson coefficient $\tilde{g}$, similarly to $C_{9(10)}\left(q^{2}\right)$, does not run at the leading order in QCD, since its colour part is simply a vector current [63].

To facilitate a comparison with the existing literature, we further define the dimensionless coupling of $V$ to the $b-s$ current, $g_{V b s}$, as

$$
g_{V b s} \equiv-\tilde{g} q^{2} .
$$

The typical size of $g_{V b s}$ is shown in figure 2 for representative choices of the input parameters. For illustration purposes, we have set $q^{2}$ to the indicative scale of $3.5 \mathrm{GeV}^{2}$. Note, however, that in the phenomenological analysis of section 4 we integrate eq. (2.10) over all the relevant invariant-mass bins.

Equation (2.10) shows that the penguin-generated Wilson coefficients depend on $q^{2}$ and the size of the width of the new gauge boson. They enter nontrivially in the calculation of the flavour observables and relative constraints when the $V$ mass lies in the vicinity of the experimental bins. The corresponding expressions should thus be compared directly to the experimental data, as we do in the numerical scan presented in section 4 . We can nonetheless provide a rough estimate of the typical size required for the dimensionful coupling $\tilde{g}$ in the limiting cases when $m_{V}$ becomes much larger or much smaller than the experimental energy (for the LFUV observables we indicatively consider this to be $q^{2} \approx 0.04-8 \mathrm{GeV}^{2}$ ). If $m_{V} \gg 10 \mathrm{GeV}$ one obtains $C_{9}^{\mu} \lesssim-0.6$ roughly for

$$
\left|\tilde{g} g_{\mu}^{V}\right| \gtrsim 1 \times 10^{-10} \frac{m_{V}^{2}}{\mathrm{GeV}^{4}}
$$




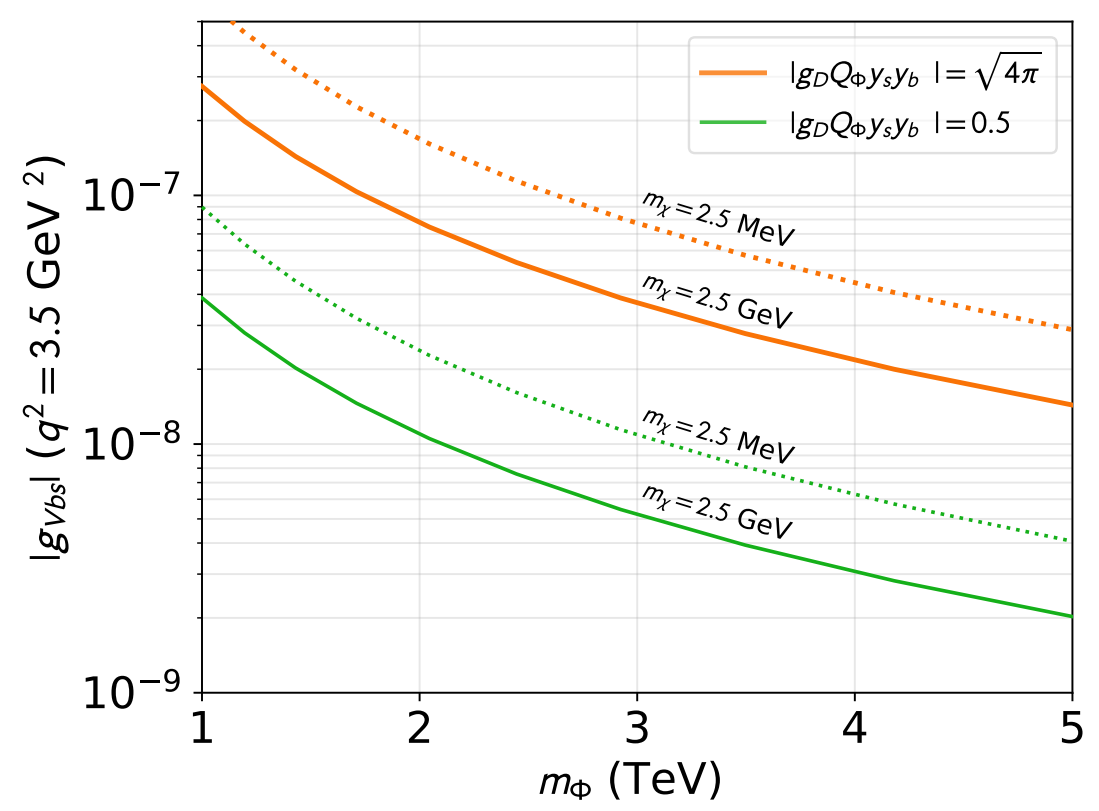

Figure 2. The typical size of $g_{V b s}$ as a function of the heavy mass $m_{\Phi}$ for different choices of the effective coupling $\left|g_{D} Q_{\Phi} y_{s}^{i *} y_{b}^{i}\right|$ given here for one light dark fermion, $\chi_{1}$. Solid (dashed) lines correspond to $m_{\chi_{1}}=2.5 \mathrm{GeV}(\mathrm{MeV})$. Indicative momentum transfer is set at $q^{2}=3.5 \mathrm{GeV}^{2}$.

where we have set $q^{2}$ at the mean momentum transfer for the $1-6 \mathrm{GeV}^{2}$ bin of the $R_{K^{(*)}}$ observables.

The two couplings $\tilde{g}$ and $g_{\mu}^{V}$ are independently constrained by two powerful probes. On the one hand, the measured $2 \sigma$ bound on $R_{B B}$ from $B_{s}$ mixing [52] has a direct impact on $\tilde{g}$ when the vector $V$ is exchanged in the $s$-channel: ${ }^{3}$

$$
|\tilde{g}| \lesssim 7 \times 10^{-8} \frac{m_{V}}{\mathrm{GeV}^{3}},
$$

where the characteristic experimental scale coincides with the $B_{s}$ mass, $q^{2}=M_{B_{s}}^{2}$.

On the other hand, the already mentioned $2 \sigma$ upper bound on the anomalous magnetic moment of the muon constrains $g_{\mu}^{V}$ to

$$
\left|g_{\mu}^{V}\right| \lesssim 7 \times 10^{-3} \frac{m_{V}}{\mathrm{GeV}}
$$

The current bounds thus result in a very narrow window of availability for explaining the flavour anomalies with penguin diagrams and $m_{V} \gg 10 \mathrm{GeV}$.

At the opposite side of the spectrum, $m_{V}<200 \mathrm{MeV}$, the new gauge boson is much lighter than the experimental energy scale. Equation (2.10) shows that the Wilson coefficients become independent of $m_{V}$ and of $q^{2}$. $C_{9}^{\mu} \lesssim-0.6$ requires approximately

$$
\left|\tilde{g} g_{\mu}^{V}\right| \gtrsim 5 \times 10^{-10} \mathrm{GeV}^{-2} .
$$

\footnotetext{
${ }^{3}$ Note, however, that $g_{V b s}$ depends directly on the product $y_{s}^{i *} y_{b}^{i}$, which produces box diagrams that are also contributing to $B_{s}$ mixing, and might result on a stronger bound than the one derived by $s$-channel exchange of $V$. We will come back to this point in section 3 .
} 
The most severe constraint in this mass range comes again from the $2 \sigma$ upper bound on the anomalous magnetic moment of the muon, which requires

$$
\left|g_{\mu}^{V}\right| \lesssim 0.6-1 \times 10^{-3}
$$

where the upper value refers essentially only to the region $m_{V} \gtrsim 100 \mathrm{MeV}$, and the lower value to all other masses below that. In light of eqs. (2.19) and (2.20) one needs $|\tilde{g}| \gtrsim$ $10^{-6} \mathrm{GeV}^{-2}$ to fit the flavour anomalies. As one can see in figure 2, effective couplings of this size are not easy to obtain in the penguin setup.

It is well known (cf., e.g., ref. [33]) that one can increase the size of $g_{\mu}^{V}$ while respecting the $2 \sigma$ upper bound on $(g-2)_{\mu}$ by introducing the axial-vector coupling $g_{\mu}^{A}$ and thus creating a negative contribution to $(g-2)_{\mu}$ that has to be fine-tuned. This, however, also induces an extremely strong contribution to the $B_{s} \rightarrow \mu \mu$ decay rate, and will prove ultimately impracticable, as will appear clear in the next sections.

Altogether, this discussion leads us to conclude that the most natural solutions are likely to be situated inside the window $m_{V} \approx 0.2-10 \mathrm{GeV}$. The remainder of this paper is thus dedicated mostly to this mass range. Note that when $m_{V}$ approaches $M_{B}$, additional resonant enhancement can be obtained to open up the parameter space, albeit at the cost of additional limits from $B \rightarrow K$ processes, which we will describe in detail in the next section.

\section{Constraints on the model}

\subsection{Flavour constraints}

$\boldsymbol{B}_{\boldsymbol{s}}$-mixing. Strong limits on the Yukawa couplings of eq. (2.5) arise from box-diagram contributions to $B_{s}$-mixing. We recall that for exclusively left-handed couplings the only relevant operator is $\mathcal{O}_{1}[40,46]$. The corresponding dimensionful Wilson coefficient is defined as

$$
C_{1}=\frac{1}{128 \pi^{2} m_{\Phi}^{2}} \sum_{i j} y_{s}^{i *} y_{b}^{i} y_{s}^{j *} y_{b}^{j} F\left(x_{i}, x_{j}\right)
$$

where the sum runs over all possible dark fermions $\chi_{i, j}$, out of which we can construct a box diagram with $\Phi$. We define $x_{i(j)}=m_{\chi_{i(j)}}^{2} / m_{\Phi}^{2}$ and the loop function reads

$$
F(x, y)=\frac{1}{(1-x)(1-y)}+\frac{x^{2} \log x}{(1-x)^{2}(x-y)}+\frac{y^{2} \log y}{(1-y)^{2}(y-x)} .
$$

In the small $x, y$ limit, the loop function can be approximated as $F(x, y) \simeq 1$, so that $C_{1} \propto 1 / m_{\Phi}^{2}$ and the limit essentially saturates. This has the unexpected consequence that, in the presence of several dark fermion states, one can readily get a large suppression of the $B_{s}$-mixing contribution in the limit where $\sum_{i j} y_{s}^{i *} y_{b}^{i} y_{s}^{j *} y_{b}^{j} \ll 1$, which can be easily obtained with, e.g., two light states $\chi_{1}, \chi_{2}$ and $y_{s}^{1 *} y_{b}^{1} \approx-y_{s}^{2 *} y_{b}^{2}$.

Note that while $C_{1}$ receives a strong suppression from the addition of approximately equal and opposite-sign couplings, there is no equivalent suppression for $C_{9}^{\mu}$, as in the limit of small $x_{1}, x_{2}$ the effective coupling $\tilde{g}$ in eq. (2.15) becomes proportional to $\ln \left(x_{1} / x_{2}\right)$. 
Limits from $\boldsymbol{B}_{\boldsymbol{s}}$ decay. When the axial-vector coupling to the muon, $g_{\mu}^{A}$, is present, the vector mediator can induce a contribution to the decay $B_{s} \rightarrow \mu^{+} \mu^{-}$via the effective operator $\mathcal{O}_{6}$, cf. eq. (2.7). (Equivalently, via the effective $b-s-V$ coupling.) The decay amplitude is expressed in terms of "effective" coefficients $C_{10}$ and $C_{P}$, whose contributions to $B_{s} \rightarrow \mu^{+} \mu^{-}$are well known [64-67].

By adopting the same convention for the scalar operator as in ref. [46] we obtain a result similar to [33]:

$$
\begin{aligned}
C_{10}\left(M_{B_{s}}^{2}\right) & =\frac{4 \pi \mathcal{N}}{\alpha_{E M}} \frac{M_{B_{s}}^{2} \tilde{g} g_{\mu}^{A}}{M_{B_{s}}^{2}-m_{V}^{2}+i m_{V} \Gamma_{V}}, \\
C_{P}\left(M_{B_{s}}^{2}\right) & =-\frac{2 M_{\mu}\left(M_{b}+M_{s}\right)}{m_{V}^{2}} C_{10}\left(M_{B_{s}}^{2}\right) .
\end{aligned}
$$

The typical bounds on $C_{P}$ are significantly more stringent than those on $C_{10}$. They are likely to have a strong impact on our results so that we include them directly in the full numerical scan present in the next section.

Limits from $B \rightarrow \boldsymbol{K}^{(*)}$ transitions. Depending on the details of the UV completion there can exist additional $B \rightarrow K^{(*)}$ decays providing strong constraints on our model. The two main limits are (1) invisible $B \rightarrow K$ transition measured by BaBar [68,69] and Belle [70, 71], $\mathcal{B R}(B \rightarrow K \nu \nu) \lesssim 1.5 \times 10^{-5}$; and (2) resonant search $B \rightarrow K^{*} V, V \rightarrow \mu \mu$ measured at $\mathrm{LHCb}$ [72], which constrains the branching ratio $\mathcal{B R}\left(B \rightarrow K^{*} V, V \rightarrow \mu \mu\right) \lesssim$ $2 \times 10^{-9}$.

Given the presence of the heavy scalar doublet $\Phi$ and one dark fermion $\chi_{1}$, one can construct the tree-level decay process $B \rightarrow K \chi_{1} \chi_{1}$ based on the $b$ quark 3-body decay $b \rightarrow \chi_{1} \Phi^{*} \rightarrow s \chi_{1} \chi_{1}$. In the limit where $m_{\chi_{1}}, M_{K} \ll M_{B}$, we have the simple expression:

$$
\Gamma_{K \chi_{1} \chi_{1}}^{\mathrm{tree}} \approx \frac{f_{+}^{2}\left|y_{s}^{1}\right|^{2}\left|y_{b}^{1}\right|^{2}}{1536 \pi^{3}} \frac{M_{B}^{5}}{m_{\Phi}^{4}}
$$

where $f_{+}^{2} \approx 0.3$ is the average value of the form factor over the range of integration of the differential decay rate. This typically leads to the constraint $\left|y_{s}^{1 *} y_{b}^{1}\right| \lesssim 10^{-2}\left(m_{\Phi} / \mathrm{TeV}\right)^{2}$ on the Yukawa couplings of any new fermion with mass $2 m_{\chi_{1}}<M_{B}-M_{K}$. As eqs. (2.8), (2.15) show, the upper bound on the Yukawa couplings strongly limits the available range of $g_{V b s}$, even when the gauge coupling $g_{D}$ is large. This means that to fit the flavour observables one has to resort to a large $g_{\mu}^{V}$ value, which, as we shall see in section 4, is severely constrained by $Z$-lineshape bounds (in addition to requiring a fine tuning of the axial-vector contribution to avoid exceeding $\left.\delta(g-2)_{\mu}\right)$. To avoid these problems the minimal particle content will have to include at least one dark fermion with mass $m_{\chi_{1}}>\left(M_{B}-M_{K}\right) / 2$.

On the other hand, there is a case to be made for the presence of additional light states with mass below the $\left(M_{B}-M_{K}\right) / 2$ threshold and Yukawa couplings to $\Phi$ and the $b$ and $s$ quarks that are small enough to avoid the tree-level $B \rightarrow K+$ inv. bound.

- Dark matter. For a fermion $\chi_{1}$ of mass above the $\left(M_{B}-M_{K}\right) / 2$ threshold the direct $s$-wave annihilation channel, $\chi_{1} \chi_{1} \rightarrow V \rightarrow \mu^{+} \mu^{-}$, is strongly constrained by CMB 
bounds [73]. Introducing one additional lighter state $\chi_{0}$ with the same quantum numbers provides instead a viable candidate for forbidden dark matter (we come back to this point in more detail at the end of section 4)

- The presence of additional light states directly affects the total width of the gauge boson $V$, potentially opening up additional parameter space for a solution to the flavour anomalies.

In cases where at least one light state $\chi_{0}$ appears besides $\chi_{1}$, two qualitatively different regimes of applicability should be considered for the invisible $B \rightarrow K$ transition. For fermions not very light and $m_{V}<2 M_{\mu}<M_{B}-M_{K}<2 m_{\chi_{i=0,1}}$, on-shell decay $B \rightarrow K V$ occurs, in which $V$ escapes undetected. It is typically suppressed in the low $m_{V}$ limit due to the momentum dependence of the effective coupling $g_{V b s}$ defined in eq. (2.15). We get

$$
\Gamma_{K V}=f_{+}^{2}\left(m_{V}\right) \tilde{g}^{2} \frac{m_{V}^{2} M_{B}^{3}}{64 \pi} \lambda\left(1, x_{K}, x_{V}\right)^{3 / 2}
$$

where we have assumed $m_{V}, M_{K} \ll M_{B}, f_{+}(0) \simeq 0.3$ is a form factor (the full expression, used in the numerical analysis of section 4 , can be found in ref. [74]), and $\lambda(x, y, z)=(x-$ $y-z)^{2}-4 x y$ is the standard Källén (triangle) function with $x_{K}=M_{K}^{2} / M_{B}^{2}, x_{V}=m_{V}^{2} / M_{B}^{2}$.

On-shell decays $B \rightarrow K \chi_{0} \chi_{0}$, occurring when $2 m_{\chi_{0}}<m_{V}<M_{B}-M_{K}$, require eq. (3.6) to be multiplied by the branching ratio $\mathcal{B R}\left(V \rightarrow \chi_{0} \chi_{0}\right)$. An additional channel is opened via the exchange of a virtual $V$ and it can dominate the invisible decay width when $m_{V}$ is small and the dark coupling $g_{D}$ is large. The full width reads

$$
\Gamma_{K \chi_{0} \chi_{0}}=\int_{4 M_{\mu}^{2}}^{\left(M_{B}-M_{K}\right)^{2}} d s \frac{\sqrt{s}}{\pi} \frac{\Gamma_{V \rightarrow \chi_{0} \chi_{0}}(s) \Gamma_{K V}(s)}{m_{V}^{2} \Gamma_{V}^{2}+\left(m_{V}^{2}-s\right)^{2}},
$$

where $\Gamma_{K V}(s)$ and $\Gamma_{V \rightarrow \chi_{0} \chi_{0}}$ are obtained by replacing $m_{V}$ by $\sqrt{s}$ in eq. (3.6) and in the corresponding $V$ decay width to fermions, and $\Gamma_{V}$ is the total width of $V$, cf. eq. (2.12). Note that with more than one dark fermion in the spectrum one ought to sum over all individual off-shell contributions.

Altogether, the combination of both real and virtual contribution to the $B \rightarrow K \chi_{0} \chi_{0}$ decay implies a complex kinematic shape in terms of missing energy, which may differ significantly from the SM-like $B \rightarrow K \nu \nu$ decay. This has the direct consequence that the experimental results of Belle and BaBar, which are optimised for the neutrino process, should not be directly applied to our scenario. We therefore perform a conservative recasting of these analyses, described in detail in appendix $\mathrm{A}$.

Finally, in the mass regime $m_{V}>2 M_{\mu}$, the light vector state can directly decay into a muon pair and this opens up the resonant channel $B \rightarrow K^{*} V, V \rightarrow \mu \mu$. This is especially important if one restricts the analysis to the case with only one dark fermion $\chi_{1}$ with $2 m_{\chi_{1}}>M_{B}-M_{K}$. The typical decay width into $K^{*}$ is given by

$$
\Gamma_{K^{*} V}=\frac{\tilde{g}^{2} m_{V}^{2} M_{B}^{5}}{64 \pi M_{K^{*}}^{2}\left(1-x_{K^{*}}\right)^{2}} \sqrt{\lambda\left(1, x_{K^{*}}, x_{V}\right)} \mathcal{F}_{1}\left(x_{K^{*}}, x_{V}\right),
$$




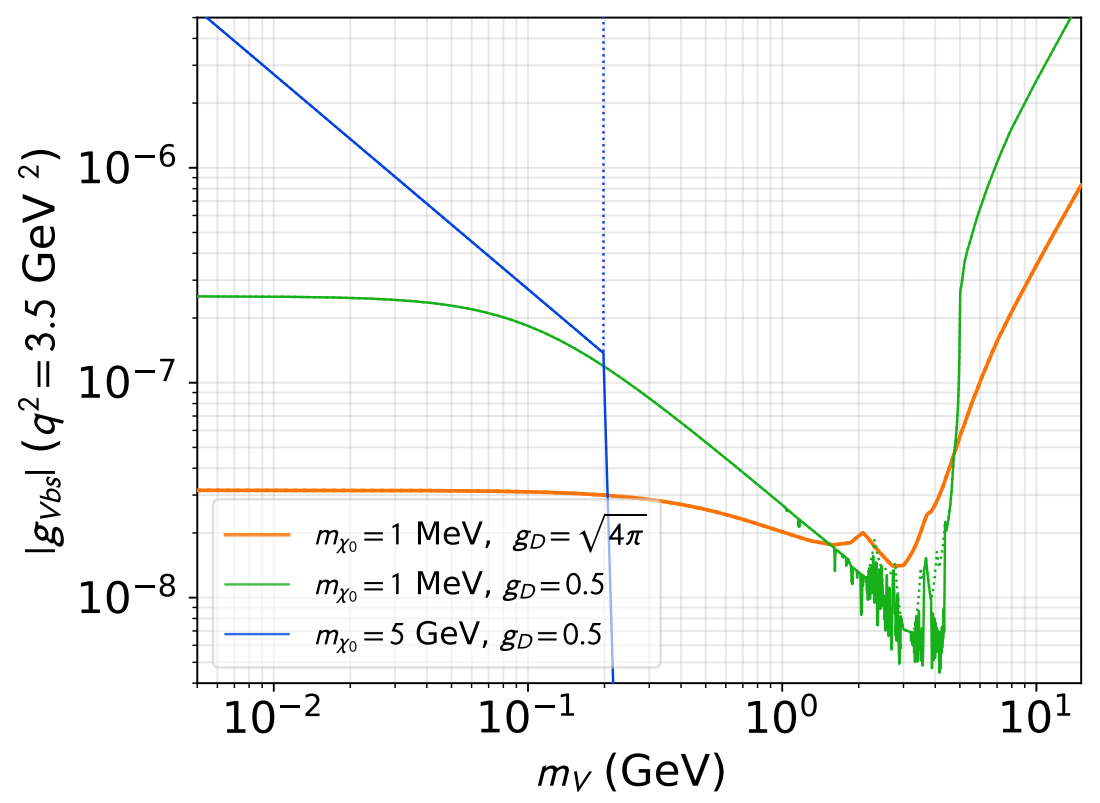

Figure 3. Upper bound on $\left|g_{V b s}\left(3.5 \mathrm{GeV}^{2}\right)\right|$ as a function of $m_{V}$ for various choices of the input parameters. We set $Q_{\Phi}=1$. The specific impact of the different constraints is described in the main text.

where an explicit expression for $\mathcal{F}_{1}\left(x_{K^{*}}, x_{V}\right)$ can be found in appendix B of ref. [35]. Note that in the limit where $m_{V} \ll M_{B}, \mathcal{F}_{1}(x, y)$ simplifies to $\mathcal{F}_{1}(x, y) \simeq 0.1 \sqrt{x}(y+1.2 x)$, so that $\Gamma_{K^{*} V}$ is typically suppressed compared to $\Gamma_{K V}$. Furthermore, the branching ratio to muons is inversely proportional to the total width $\Gamma_{V}$ and can thus be strongly suppressed if the coupling of $V$ to extra dark fermions $\chi_{i}$ is large.

Note that the limits from LHCb [72] on this process focused on a narrow, or even long-lived, new resonance, with limits based on invariant mass bins of a few MeV. This hypothesis is especially problematic when the mediator is around the $\mathrm{GeV}$ range, since a large dark gauge coupling implies a very large width for $V$. We then simply model the resonance via a Breit-Wigner distribution and compare bin-by-bin with the limit of ref. [72], retaining the strongest bin as the main limit. ${ }^{4}$

The overall impact of the limits from $B \rightarrow K^{(*)}$ transitions on the size of the effective coupling $g_{V b s}$ is summarised in figure 3 as a function of the vector mass $m_{V}$, for three representative choices of the pair $\left(m_{\chi_{0}}, g_{D}\right)$. For $m_{\chi_{0}}=5 \mathrm{GeV}$, the decay $B \rightarrow K \chi_{0} \chi_{0}$ is kinematically forbidden, so that invisible $B$ decays can only proceed via the on-shell process $B \rightarrow K V$. This results in a weak bound in the small $m_{V}$ regime (blue line). Note that when $m_{V}>2 M_{\mu}$, the constraints from resonant searches using $B \rightarrow K^{*} \mu \mu$ strongly exclude this setup since $V \rightarrow \mu \mu$ is in this case the only accessible decay channel.

\footnotetext{
${ }^{4}$ While it is clear that a complete recasting of the LHCb analysis [72] for a large-width NP signal would impact the $\sim \mathrm{GeV}$ mass range of $V$, we do not expect significant modifications to the overall picture discussed in section 4 since: (1) limits on $\mathcal{B R}(B \rightarrow K+$ inv.) already forbid scan points compatible with the flavour anomalies in this region when the invisible width is large; (2) this region has a strong background from the charm-quark resonances [33]; and (3) we already include the main flavour observable in the numerical scan via the differential branching ratio of $B \rightarrow K \mu \mu$.
} 
In the presence of dark fermions with a small mass, $2 m_{\chi_{0}}<M_{B}-M_{K}$, the decay channel $V \rightarrow \chi_{0} \chi_{0}$ opens up and the relative strength of different bounds depends on the size of $g_{D}$. In the case of large $g_{D}$ (orange line), if $2 m_{\chi_{0}}<m_{V}<M_{B}-M_{K}$ both the on-shell and off-shell invisible decays contribute to the width. For light vector mediator the off-shell decay takes over due to the $q^{2}$ dependence of the coupling and the bound on $g_{V b s}$ saturates. If, on the other hand, $g_{D}$ is smaller (green line) and $m_{V}>2 M_{\mu}$, the constraints from resonant searches using $B \rightarrow K^{*} \mu \mu$ typically overcome the invisible decay limits, suppressing $g_{V b s}$ by an order-one factor.

Finally, note that for large $m_{V}$ the limit arises from invisible searches from the off-shell decay $B \rightarrow K \chi_{0} \chi_{0}$ when it is kinematically allowed.

\subsection{Precision physics constraints}

Muon anomalous magnetic moment. The couplings of $V$ to muons can be constrained by the measurement of the anomalous magnetic moment of the muon. A contribution to $(g-2)_{\mu}$ is in this case given by $[75,76]$

$$
\delta(g-2)_{\mu}=\frac{1}{8 \pi^{2}} \frac{M_{\mu}^{2}}{m_{V}^{2}} \mathcal{F}\left(\frac{M_{\mu}}{m_{V}}\right)
$$

where

$$
\mathcal{F}(x)=\int_{0}^{1} d z \frac{\left(g_{\mu}^{V}\right)^{2} 2 z^{2}(1-z)+\left(g_{\mu}^{A}\right)^{2}\left[2 z(1-z)(z-4)-4 x^{2} z^{3}\right]}{x^{2} z+(1-z)\left(1-x^{2} z\right)} .
$$

As was mentioned in section 2, given the limited range achievable in penguin constructions for $g_{V b s}$, when only vector-like couplings to the muons are present it becomes difficult to find a $g_{\mu}^{V}$ value large enough to allow for a reasonable agreement with the flavour anomalies and at the same time not too large a deviation from the measured value of $(g-2)_{\mu}$. A certain level of cancellation with the contribution from the axial-vector coupling must take place in most situations [33]. For a GeV-scale vector mediator, this occurs for $g_{\mu}^{A} \approx-0.44 g_{\mu}^{V}$. Note, however, that including the axial-vector contribution triggers the strong bounds from $B_{s} \rightarrow \mu \mu$ discussed above.

$Z$ physics and intensity frontier limits. The coupling of the $Z$ boson to the muon is modified at the one-loop level $[77,78]$ within UV constructions of the type as in Footnote 2. However, due to the smallness of the $Z$-boson coupling to charged leptons in the SM, the limit is typically subdominant with respect to the $(g-2)_{\mu}$ bound.

A powerful method for discerning light resonances through precision measurements of Drell-Yan dimuon production was proposed in ref. [79]. For $m_{V}=1-5 \mathrm{GeV}$ an upper bound can be derived

$$
\sqrt{\left(g_{\mu}^{V}\right)^{2}+\left(g_{\mu}^{A}\right)^{2}} \lesssim 5.6 \times 10^{-2}\left(1+0.13 \frac{m_{V}}{\mathrm{GeV}}\right)
$$

Finally, the Belle-II Collaboration recently provided a bound on the final state radiation process $e^{+} e^{-} \rightarrow \mu^{+} \mu V, V \rightarrow$ invisible, based on $0.28 \mathrm{fb}^{-1}$ of data from the 2018 
run [80], which applies directly to our model. While the current limit can hardly compete with the Drell-Yan bound, the 2019 run has stored $\sim 10 \mathrm{fb}^{-1}$ and moreover a few $\mathrm{ab}^{-1}$ should be obtained in 2020, so that future data will become rapidly relevant.

Notice that more generically, for a $V$ mass above $\sim 10 \mathrm{GeV}$, the phenomenology of the light fermions in intensity frontier experiments can be obtained by integrating it out and considering the fermion portal four-fermion operators [81]. Similarly, the limit from the tree-level $b \rightarrow \chi \Phi^{*} \rightarrow s \chi \chi$ decay can also be obtained by integrating out the heavy scalar $\Phi$ and using the existing bounds on the fermion portal operator $\bar{b} \gamma^{\mu} s \bar{\chi} \gamma_{\mu} \chi$. While we cover directly the relevant limits in this section, the latter approach could be particularly fruitful to study and constrain the possible couplings between new light fermions and the other SM generations.

Neutrino trident production. If the gauge boson features a coupling $g_{\nu}$ to muon neutrinos (cf. section 2.2), one expects a strong enhancement in the neutrino trident production from scattering on atomic nuclei, $N: N \nu \rightarrow \nu N \mu^{+} \mu^{-}$[59].

The cross section for this process has been measured by the CCFR [60] and CHARMII [61] collaborations to be in agreement with the SM prediction. In the range $m_{V}>1 \mathrm{GeV}$ it results in the generic bound

$$
g_{\nu} \lesssim 0.002\left(\frac{m_{V}}{\mathrm{GeV}}\right)
$$

which roughly saturates for smaller mass to about $g_{\nu} \lesssim 0.001$.

When the bound appears in the plots of section 4 it is obtained under the assumption $g_{\nu} \equiv g_{\mu}^{V}$ (we repeat that whether or not the bound is relevant depends on the UV completion).

Kinetic mixing. In presence of states charged both under the $\mathrm{U}(1)_{Y}$ and $\mathrm{U}(1)_{D}$ symmetry groups, kinetic mixing $\epsilon$ between the photon and the vector $V$ will be generated at the loop level. The corresponding 1-loop contributions from fermions and scalars are given by

$$
\epsilon \approx \frac{g_{D} g_{Y} \cos \theta_{W}}{12 \pi^{2}}\left(\sum_{f \in \text { ferm. }} N_{3_{f}} Y_{f} Q_{f}+\frac{1}{8} \sum_{s \in \text { scal. }} N_{3_{s}} Y_{s} Q_{s}\right),
$$

where $Y_{f, s}$ is the fields' hypercharge, $Q_{f, s}$ is the dark charge, and the coefficients $N_{3 f, s}$ indicate the dimension of the $\mathrm{SU}(3)_{c}$ representation.

The fields that contribute to the kinetic mixing are $\Phi, \mu_{L}$, and $\mu_{R}$ which, when $g_{\mu}^{V, A} \ll$ $g_{D}$, results in

$$
\epsilon \approx 8 \cdot 10^{-4} \times\left(\frac{g_{D}}{3}\right)
$$

Such a kinetic mixing is at the limit of exclusion given the current intensity frontier searches (see, e.g., ref. [82] for a recent review), especially when invisible decay channels are not available for the vector mediator. However, the precise value of the kinetic mixing is strongly dependent on the UV physics, and additional VL fields can modify the prediction of eq. (3.13) although not by many orders of magnitude. 


\begin{tabular}{|c|c|}
\hline Parameter & Prior range \\
\hline$|r|$ & {$[0.075,25]$} \\
$g_{\mu}^{V}$ & {$[0.001,0.2]$} \\
$\gamma_{V}^{D}$ & {$[0.001,0.4]$} \\
\hline$r_{A V}$ & {$[-1,0]$} \\
$m_{V}(\mathrm{GeV})$ & {$[0.6,2]$ and $[2,15]$} \\
\hline
\end{tabular}

Table 1. Prior ranges for the free parameters of the model. For the first three parameters the prior distribution is given in logarithmic scale, while for the others it is given in linear scale.

LHC constraints on $\boldsymbol{t} \rightarrow \boldsymbol{c} \boldsymbol{\mu} \boldsymbol{\mu}$. We work in this paper under the assumption that the only nonzero Yukawa couplings of the down-like type are $y_{s}^{i}, y_{b}^{i}$. However, as eq. (2.5) shows, the corresponding Yukawa couplings of the up-like type, $y_{c}^{i}$, $y_{t}^{i}$, do not receive CKM suppression. They can thus generate non-negligible contributions to processes involving $t \rightarrow c \mu \mu$ transitions.

Effective operator analyses of LHC bounds from rare top decays [83, 84], derived originally for the very-high mass regime, $m_{V} \sim \mathcal{O}(\mathrm{TeV})$, impose a fairly weak bound on the coupling product when $m_{V} \ll M_{t}$ :

$$
\left|\tilde{g} g_{\mu}^{V}\right| \lesssim 10^{-6} \mathrm{GeV}^{-2} .
$$

A rough comparison with eq. (2.16) shows that this is not likely to be constraining for our scenarios. However, given that eq. (2.10) presents a nontrivial $q^{2}$ dependence, for the light mass range investigated in this paper one should rather perform a detailed recast of the experimental searches. This task exceeds the purpose of the present paper in view of the fact that, as we will show in section 4 , the flavour and intensity frontier experiments discussed above provide already a set of powerful and often inescapable constraints on the dark sector.

\section{$4 \quad$ Fitting procedure and results}

Fitting procedure. We perform a multidimensional fit of the following free parameters: $m_{V}, g_{\mu}^{V}, r_{A V} \equiv g_{\mu}^{A} / g_{\mu}^{V}, \gamma_{V}^{D}, r=g_{D} Q_{\Phi} y_{s}^{1 *} y_{b}^{1}$ (with only one light dark fermion $\chi_{1}$ contributing with Yukawa couplings $y_{s}^{1}$ and $\left.y_{b}^{1}\right) . r$ is employed here as a proxy for the effective coupling $\tilde{g}$ once the mass parameters are fixed. We choose $m_{\chi_{1}}=2.5 \mathrm{GeV}$ and $m_{\Phi}=1 \mathrm{TeV}$; under these assumptions $r$ relates to $\tilde{g}$ as $|\tilde{g}|=\left|2.2 \times 10^{-8} \mathrm{GeV}^{-2} r\right|$. Since the fitted flavour observables depend on $\tilde{g}$ rather than the couplings composing it, our results can be extended straightforwardly to the case with more light fermions.

The prior ranges of the fitted parameters are presented in table 1. Separate fits are performed depending on whether $m_{V}$ lies above or below the relevant bins for the $B$ anomalies. In the text we refer to these sets as the high-mass fit and the low-mass fit. This is required by the fact that in order to obtain a negative $C_{9}^{\mu}$ the product $r \cdot g_{\mu}^{V}$ should assume a different sign in each of these two regions (we will come back to this point 
later on, when discussing our numerical results). We observe that $m_{V}=2 \mathrm{GeV}$ gives an approximate threshold separating the two regimes.

We fit the free parameters of the model to the available experimental data reporting anomalies in $B$-meson decays, namely the LFUV ratios $R_{K}, R_{K^{*}}$, the angular observables in the $B \rightarrow K^{*} \mu^{+} \mu^{-}$decay and the branching ratio of $B_{s} \rightarrow \mu \mu .^{5}$ In the high-mass regime we include in the likelihood function the experimental $2 \sigma$ upper bound on the anomalous magnetic moment of the muon, $\delta(g-2)_{\mu} \lesssim 4 \times 10^{-9}$. We do not incorporate this bound in the likelihood function of the low-mass fit to avoid driving the scan too forcefully towards parameter space regions that are in tension with the remaining constraints.

To carry out the fit, we employ the HEPfit package [85], performing a Markov Chain Monte Carlo (MCMC) analysis by means of the Bayesian Analysis Toolkit (BAT) [86]. A set of $\mathcal{O}(500 K)$ points is generated for the two scenarios described in table 1 , and for each case the subset of points reproducing the $B$ anomalies, $\mathcal{B R}\left(B_{s} \rightarrow \mu \mu\right)$, and the upper $(g-2)_{\mu}$ bound (in the high-mass case only) at the $2 \sigma$ level, is stored. These initial subsets of points are then subjected to additional constraints coming from $B_{s}$ mixing, Drell-Yan production and, when applicable, $B \rightarrow K+$ inv. searches.

Results for high-mass. We start with addressing solutions in the high-mass range, $m_{V}=2-15 \mathrm{GeV}$.

Two dark fermions in the theory: $m_{\chi_{0}} \leq m_{\chi_{1}}$. We describe here a case in which the gauge boson $V$ features a non-negligible invisible width, which could stem from the presence in the spectrum of an additional light fermion $\chi_{0}$ besides $\chi_{1}$ (see discussion in section 3.1). The results of the scan in the $\left(m_{V}, g_{\mu}^{V}\right)$ plane are presented in figure 4 . The yellow points are obtained in the scanning procedure described above and correspond to the models in which the $B$ anomalies, $\mathcal{B R}\left(B_{s} \rightarrow \mu \mu\right)$, and $\delta(g-2)_{\mu}$ are fitted at the $2 \sigma$ level. The green points are those that remain allowed after the limits from $B \rightarrow K+$ inv. transitions are applied, following the recasting procedure outlined in appendix A.

The on-shell process $B \rightarrow K V, V \rightarrow \chi_{0} \chi_{0}$ typically proceeds unsuppressed for a $\mathrm{GeV}$ scale mediator, so that all the yellow points below the $M_{B}-M_{K}$ threshold are excluded. On the other hand, the limits on $\tilde{g}$ are dramatically weakened above the $M_{B}-M_{K}$ threshold (see figure 3 ), so that in this regime one can easily fit simultaneously $(g-2)_{\mu}$ and the flavor anomalies. Incidentally, we find that solutions to the flavour anomalies with $m_{V} \approx 2.5 \mathrm{GeV}$, corresponding to the case described in ref. [33], are excluded. The reason is that, unlike ref. [33], in our framework $g_{V b s}$ is $q^{2}$-dependent and induces an $\left(m_{V} / \mathrm{GeV}\right)^{2}$ enhancement to the size of eq. (3.6).

An additional bound on the parameter space of the model is derived from the $Z$ lineshape from Drell-Yan at the LHC, which strongly affects the maximal allowed value of $g_{\mu}^{V}$ [79]. The corresponding exclusion region is depicted in figure 4 in dark grey. The shading is obtained under the assumption $g_{\mu}^{A}=-0.44 g_{\mu}^{V}$, a relation that induces destructive

\footnotetext{
${ }^{5}$ Due to the explicit $q^{2}$ dependence of $C_{9,10}^{\mu}$ it is not possible to match the Wilson coefficients to the model-independent bounds obtained by the global fits in the literature. Instead, $C_{9,10}^{\mu}$ have to be fitted directly to the experimental data. On the other hand, if $m_{V}$ is in the $\mathrm{MeV}$ range, it is possible to directly match the Wilson coefficients to the model-independent bounds obtained by the global fits.
} 


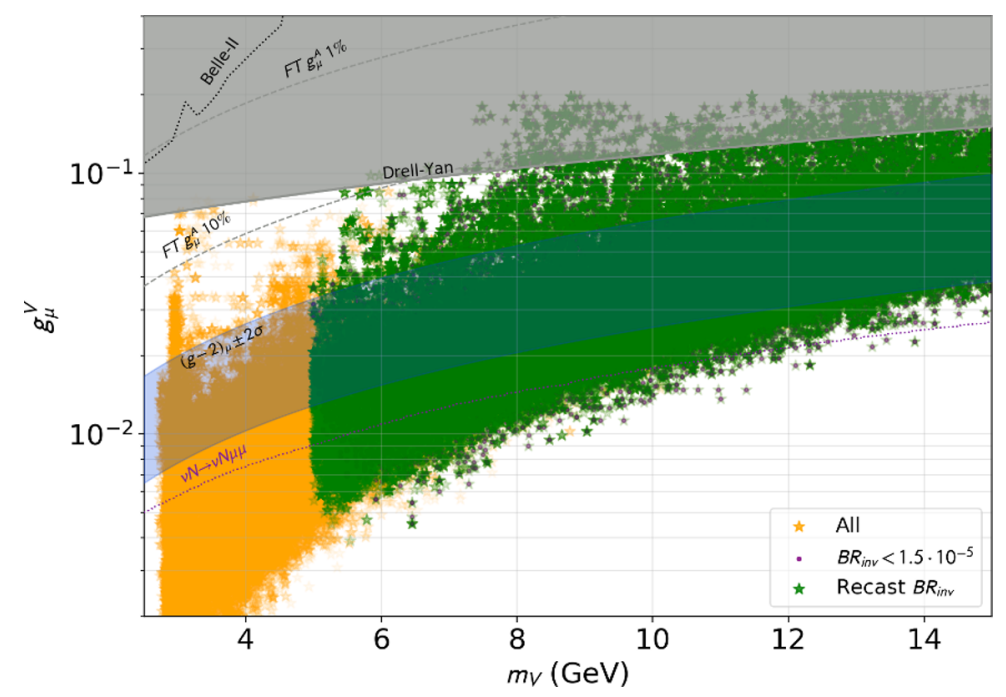

Figure 4. In yellow model points in which the $b \rightarrow s$ anomalies, $\mathcal{B R}\left(B_{s} \rightarrow \mu \mu\right)$, and $(g-2)_{\mu}$ are fitted at the $2 \sigma$ level. Superimposed points are allowed after the limits from $B \rightarrow K+$ inv. are applied, as reported by CLEO [87] (purple) or using our recast procedure (green), cf. appendix A. Grey shaded region is excluded by precision measurements of Drell-Yan at the LHC [79]. Purple dotted line indicates the exclusion bound from the neutrino trident production [59]. Dotted black line indicates the upper bound from Belle II [80]. In light blue we mark the region in which the $(g-2)_{\mu}$ constraint is satisfied at $2 \sigma$ with $g_{\mu}^{A}=0$.

interference in the calculation of $(g-2)_{\mu}$ (cf. eqs. (3.9) and (3.10)). It is well known [33] that the above relation between the vector and axial-vector coupling requires some level of fine tuning. The grey dashed lines in figure 4 trace the value of $g_{\mu}^{V}$ that corresponds to the indicated level of fine tuning in $g_{\mu}^{A} \approx-0.44 g_{\mu}^{V}$ required to avoid exceeding the $2 \sigma$ upper bound from the measurement of $\delta(g-2)_{\mu}$. Note, however, that the tuning of the vector and axial-vector muon couplings is a priori not needed in the high-mass regime, as confirmed by the large number of green points within the blue shaded band, corresponding to the region satisfying the $(g-2)_{\mu}$ constraint at $2 \sigma$ with $g_{\mu}^{A}=0$. This is an attractive feature of our model, in which we can obtain relative low values of $g_{\mu}^{V}$ and subsequently avoid the Drell-Yan limit.

In figure 4 the limits from the neutrino trident production derived in ref. [77] for $g_{\mu}^{A}=0$ are shown as a dashed purple line. The constraint applies only if the mediator couples directly to neutrinos, cf. discussion in section 2.2. It should be stressed that, even when the neutrino trident bound applies, solutions that escape the experimental limit exist, with $m_{V}=5-14 \mathrm{GeV}$ and $r$ at the upper end of the scanned range.

Finally, it is instructive to compare the results obtained within our UV-complete setup to those derived in simplified $Z^{\prime}$ models with an effective coupling to the $b-s$ current, $g_{b s}$. For example, a solution to the $b-s$ anomalies (without the $(g-2)_{\mu}$ constraint) was found in ref. [18], with $m_{Z^{\prime}}=10 \mathrm{GeV}, g_{\mu}^{V}=-g_{\mu}^{A} \approx 10^{-2}$, and $g_{b s} \approx 5 \times 10^{-6}$. Figure 4 shows that we obtain solutions characterised by similar mass and muon coupling in our setup, corresponding, again, to $r$ values at the upper end of the scanned range. 


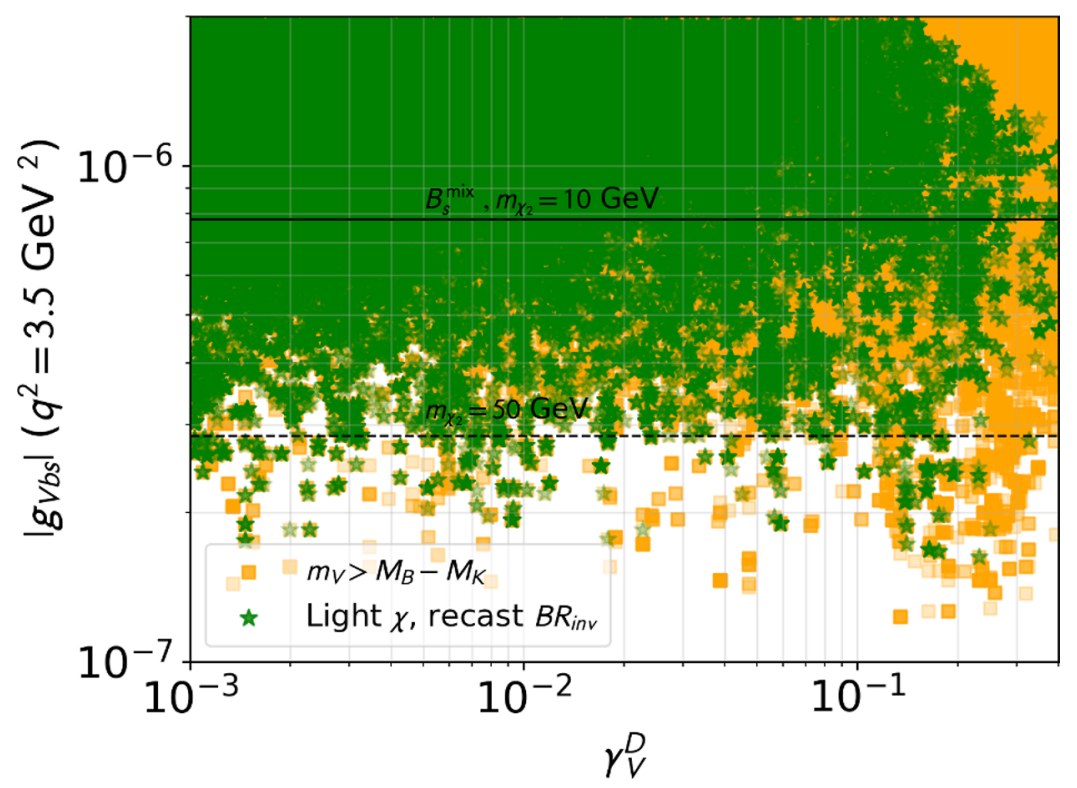

Figure 5. Results of the scan in the high-mass region, $m_{V}=2-15 \mathrm{GeV}$, in the plane of the effective coupling $\left|g_{V b s}\right|$ at $q^{2}=3.5 \mathrm{GeV}^{2}$ versus $\gamma_{V}^{D}$. All points in the plot satisfy the flavour constraints at $2 \sigma$. Yellow squares have $m_{V}>M_{B}-M_{K}$ by default, while green stars lie on a broader mass range but must survive the $B \rightarrow K+$ inv. limit. The latter is obtained from our recasting procedure assuming $V$ has a $\sim 100 \%$ decay rate into a light dark fermion with $m_{\chi_{0}} \ll\left(M_{B}-M_{K}\right) / 2$. Horizontal grey lines represent the upper bound from $B_{s}$ mixing on the effective coupling, under the assumption $y_{s}^{1 *} y_{b}^{1}+y_{s}^{2 *} y_{b}^{2}=0$ for two different mass values of the compensating dark fermion: $m_{\chi_{2}}=10 \mathrm{GeV}$ (solid) and $m_{\chi_{2}}=50 \mathrm{GeV}$ (dashed).

One dark fermion $\chi_{1}$ in the theory. To conclude the discussion of figure 4, we point out that the picture does not receive substantial modifications if the light fermion $\chi_{0}$ is not introduced in the theory, and the only NP fermion sits at $m_{\chi_{1}}=2.5 \mathrm{GeV}$. In this case, the $B \rightarrow K+$ inv. bound does not apply. However, all points with mass $m_{V}<M_{B}-M_{K}$ become subject to the strong resonant $B \rightarrow K^{*} \mu \mu$ limit, which cuts drastically the parameter space and induces solutions not dissimilar to the area delimited in green in figure 4 .

Constraints from $B_{s}$ mixing. The parameter space shown in figure 4 has not been subject to constraints from $B_{s}$ mixing, which predominantly proceeds via box diagrams involving dark fermions and heavy coloured scalars, and which in principle could put a strong limit on the NP Yukawa couplings. On the other hand, the LFUV and angular observables in the fit depend only indirectly on $y_{b}$ and $y_{s}$, via the effective coupling $\tilde{g}$. In the presence of several light fermions, a possible way of suppressing $B_{s}$ mixing in the limit $m_{\chi_{i}} / m_{\Phi} \ll 1$ is obtained when $\sum_{i j} y_{s}^{i *} y_{b}^{i} y_{s}^{j *} y_{b}^{j} \ll 1$, even if one of the $\chi_{i}$ is relatively heavier than the others, as shown in section 3.1.

We illustrate in figure 5 the dependence of the $B_{s}$-mixing bound, which can limit the size of $g_{V b s}$, on the mass of one additional light fermion introduced in the theory as a means to cancelling the $B_{s}-B_{s}$ box diagram. We start by ploting $g_{V b s}$ as a function of the $V$ width, or rather the parameter $\gamma_{V}^{D}$ defined in Eq (2.13). Green points correspond to 


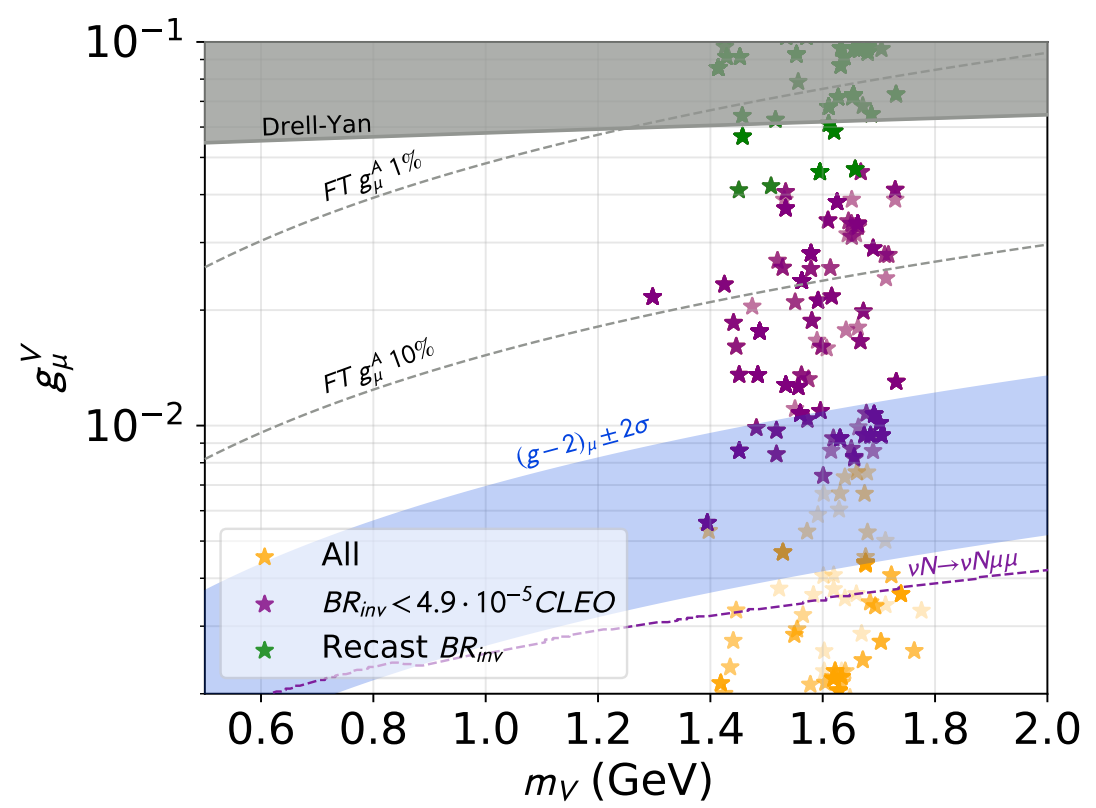

Figure 6. Results of the scan in the low $m_{V}$ region. The colour code is the same as in figure 4 .

the solutions also marked in green in figure 4, which provide a satisfying fit to the flavour observables and escape $B \rightarrow K+$ inv. limits when this channel is open. Yellow points show the corresponding case with only $\chi_{1}$ in the spectrum, which are subject predominantly to the bound from $B \rightarrow K^{*} \mu \mu$. The parameter space is partially tilted towards the small width for the green points, since the channel $B \rightarrow K+$ inv. is open and constrains the larger invisible widths.

The presence or not of the light state $\chi_{0}$ has little influence on the overall $B_{s}$-mixing constraints due to its small Yukawa couplings. On the other hand, the box-diagram induced contribution from $\chi_{1}$ would limit $g_{V b s}$ to be around $10^{-8}$, indicating the need for an additional contribution to $B_{s}$-mixing in the UV of the theory. As we have discussed previously in section 3.1, a particularly simple way out invokes the presence of an extra, more massive, dark fermion $\chi_{2}$. The solid line shows the upper bound on $g_{V b s}$ when $m_{\chi_{2}}=10 \mathrm{GeV}, g_{D}=\sqrt{4 \pi}$, and we impose $\delta_{Y}=y_{s}^{1 *} y_{b}^{1}+y_{s}^{2 *} y_{b}^{2}=0$. The corresponding limit when $m_{\chi_{2}}=50 \mathrm{GeV}$ is shown with a dashed line. Reducing the fine tuning to $\delta_{Y}=0.05$ lowers the lines by a factor of 5 .

Results for low-mass. We show in figure 6 the results of the scan for the low-mass range, $m_{V}=0.6-2 \mathrm{GeV}$, in the $\left(m_{V}, g_{\mu}^{V}\right)$ plane. Recall that to obtain this region of the parameter space one has to switch the sign in the product $r \cdot g_{\mu}^{V}$ with respect to the high-mass region. The procedure allows one to fit $R_{K^{(*)}}$ correctly by means of destructive interference with the SM value of $C_{9}^{\mu}$ below the experimental bin. The colour code in figure 6 is the same as in figure 4. Note that in this region the mediator must have a sizeable invisible width to avoid stringent constraints from a visible dimuon resonance in the $B \rightarrow K^{*}$ spectrum, as discussed in section 3.1. The presence of at least one light fermion $\chi_{0}$ besides $\chi_{1}$ is therefore given for granted. 
A few takeaways emerge from the scan in the low-mass region. The first is that there are no solutions with mass $m_{V} \lesssim 1.4 \mathrm{GeV}$. In fact, they are cut out by the $\mathcal{B R}\left(B_{s} \rightarrow \mu \mu\right)$ constraint, which is directly implemented in the likelihood function. Besides the mass cut, the surviving points are all characterised by fairly large values of $R_{K^{(*)}}$, which both lie $2-3 \sigma$ away from the central value measured at $\mathrm{LHCb}$ and closer to their SM expectation. For the same reason, the plot appears much sparser than in the high-mass case: very few model points can be found within $2 \sigma$ of the measured values of LFUV observables and $\mathcal{B R}\left(B_{s} \rightarrow \mu \mu\right)$ simultaneously. We thus identify a mild tension in this part of the parameter space.

Note also that the models surviving the bound from $B \rightarrow K+$ inv. searches (green points) require a large coupling $g_{\mu}^{V}$ to the muon, as $\tilde{g}$ is directly constrained by the invisible search. This means a large level of fine tuning in the corresponding $g_{\mu}^{A}$ value necessary to cancel $\delta(g-2)_{\mu}$. Overall, it is clear that the low-mass region is under siege from a combination of complementary bounds but at present it is not entirely excluded.

Let us finish this section by mentioning the case of a very light mediator: a new gauge boson with mass in the $\mathrm{MeV}$ range while dark fermions lie in the $\mathrm{GeV}$ scale. An interesting property of this regime is that, in the limit where $2 m_{\chi_{i}}>M_{B}-M_{K}$, the mediator $V$ is essentially long-lived since is does not have any available tree-level decay channel. The invisible $B$ decay is then driven exclusively by the $B \rightarrow K V$ process, which is strongly suppressed at low $m_{V}$. Furthermore, the dependence of the Wilson coefficients on $q^{2} /\left(q^{2}-m_{V}^{2}\right)$ converges to a constant when $m_{V} \ll q$ and it closely resembles the standard electromagnetic penguin contribution to the flavour anomalies. However, given that the limit from $B_{s} \rightarrow \mu \mu$ decay forbids such a light vector mediator to have a significant axialvector coupling to the muon, cf. eqs. (3.3), (3.4), one cannot avoid the upper bound on the vector coupling arising from $\delta(g-2)_{\mu}: g_{\mu}^{V} \lesssim 7 \times 10^{-4}$. Concretely, in order to obtain $C_{9}^{\mu} \approx-0.7$ a coupling $\tilde{g} \gtrsim 10^{-6} \mathrm{GeV}^{-2}$ is required, which can only by achieved while satisfying $B \rightarrow K+$ inv. limits if $m_{V} \lesssim 5 \mathrm{MeV}$, cf. figure 3 . A vector mediator this light is already excluded by the standard searches for long-lived dark photon. We conclude that no solution with $V$ in the $\mathrm{MeV}$ range is available in penguin-generated scenarios.

Dark matter. As an interesting aside, the lightest dark fermion can provide a good example of forbidden dark matter candidate when its mass is below the muon mass. The dominant annihilation channel for such a dark matter candidate would be $\chi_{0} \bar{\chi}_{0} \rightarrow V^{*} \rightarrow$ $\mu \bar{\mu}$. Such a process leads to a typical relic density of

$$
\Omega h^{2} \approx 0.05\left(\frac{100 \mathrm{MeV}}{m_{\chi_{0}}}\right)^{2}\left(\frac{m_{V}}{8 \mathrm{GeV}}\right)^{4}\left(\frac{0.05}{g_{\mu}^{V}}\right)^{2}\left(\frac{1}{\alpha_{D}}\right) e^{-2 \Delta \cdot x_{f}},
$$

where $x_{f} \approx 20$ for the relevant masses, $g_{D}=\sqrt{4 \pi}$ and $\Delta=1-m_{\chi_{0}} / M_{\mu}$.

When $m_{\chi_{0}}$ drops below the muon mass threshold the relic density is exponentially enhanced since the annihilation process can only occur due to the thermal velocity of the dark matter particle in the early universe [88, 89]. This ensures that the thermal target is matched for one coupling-dependent mass below $M_{\mu}$, typically around $\sim M_{\mu} / 2$. Furthermore, all other annihilation processes are exponentially suppressed when the universe 
temperature decreases, ensuring that the $\mathrm{CMB}$ limits on late-time annihilating sub-GeV dark matter are automatically escaped.

\section{Conclusions}

We have presented in this work a solution for the $b \rightarrow s$ flavour anomalies based on the presence of a split dark sector with a light vector mediator as well as new light Dirac fermions which may constitute all or part of the dark matter. The interaction with the $b$ and $s$ quarks is generated at the loop level via the addition of a coloured scalar particle, resembling a supersymmetric squark. We analysed numerically and analytically the resulting low-energy effective theory, which in particular possesses a $q^{2}$-dependent interaction of the vector mediator with $b$ and $s$ quarks. Varying the mass of the vector mediator from the $\mathrm{MeV}$ scale to the tens of $\mathrm{GeV}$, we find two scenarios satisfying all experimental constraints while providing a good fit to the anomalies. In particular, the region with a $\mathrm{GeV}$-scale mediator above the $B-K$ mass threshold appears particularly promising, requiring little to no tuning in the low-energy effective parameters, and to the best of our knowledge it has not been considered previously.

Since our model is partially embedded in a UV completion, we have additionally pointed out several constraints that can challenge its viability. We have highlighted the constraints from the $B \rightarrow K+$ inv. decay rate and $B_{s}$ mixing and, in the latter case, provided an example of a mechanism to escape it. We did not make any assumption in this paper on the nature of the dark Dirac-fermion interactions with the neutrinos. Indeed, since the former are complete SM singlets, it would be very interesting to investigate whether or not they could behave as right-handed neutrinos (for instance via the coupling to a dark charged new Higgs doublet), and in that case investigate their relationship with the strong neutrino trident limits.

While the experimental constraints on models addressing the flavour anomalies with light mediators are already quite stringent, we have identified several observables that can easily exclude these scenarios entirely or provide smoking-gun proof of their detection. Chief among those are the limits from $B \rightarrow K$ and $B \rightarrow K^{*}$ transitions. While the former play a critical role via the $B \rightarrow K+$ inv. bounds, experimental searches are typically optimised for the SM process $B \rightarrow K \nu \nu$. Including an analysis based on a light, and potentially broad, invisible resonance $B \rightarrow K V$ could likely strengthen significantly the existing limits, especially when the mediator is light. Similarly, the latest search for $B \rightarrow$ $K^{*} \mu \mu$ has focused on a very narrow resonance, and should be properly recast for the case of a large and invisible width. Finally, it is important to note that limits on a light dark photon are due to improve in the next few years, and will further constrain the case of a mediator at and below the GeV scale.

\section{Acknowledgments}

LD thanks S. Robertson, M. Heck, M. Williams and G. De Pietro for interesting discussions. We thank Pere Arnan for pointing out a factor of 2 mistake in the normalisation 
of eq. (2.8). LD is supported by the INFN "Iniziativa Specifica" Theoretical Astroparticle Physics (TAsP-LNF). MF is supported by the MINECO grant FPA2016-76005-C2-1-P and by Maria de Maetzu program grant MDM-2014-0367 of ICCUB and 2017 SGR 929. KK is supported in part by the National Science Centre (Poland) under the research Grant No. 2017/26/E/ST2/00470. EMS is supported in part by the National Science Centre (Poland) under the research Grant No. 2017/26/D/ST2/00490.

\section{A Invisible decay limits}

We present in this appendix a more detailed treatment of the recasting procedure performed to extract conservative $B \rightarrow K+$ inv. limits on our model.

While the CLEO Collaboration searched explicitly for an on-shell light particle mediating the $B \rightarrow K$ decay [87], their $2 \sigma$ limit $\mathcal{B R}(B \rightarrow K+$ inv. $)<4.9 \times 10^{-5}$ is relatively weak compared to the ones from $B$ factories. In the following we will base our limit both on the BaBar result [68] - which provides a differential branching ratio limit in bins of $q^{2}$ - as well as on an older analysis from the same collaboration [69], which also had some differential limits, albeit on a much larger range for $q^{2}$. Note that the current bounds from the Belle Collaboration [70, 71] are typically of the same order as for BaBar. They are however strongly optimised for the SM-like $B \rightarrow K \nu \nu$ signal and only present their bounds in the total integrated branching ratio. We therefore concentrate on the two BaBar analyses.

First, using the BaBar hadronic-tagging analysis [68], we calculate the branching ratio $\mathcal{B R}\left(B \rightarrow K \chi_{0} \chi_{0}\right)$ in $s_{B}=q^{2} / M_{B}^{2}$ bins, where for low $m_{V}$ most of our NP signal is concentrated in the lowest bin, $s_{B}<0.1$. We then compare it with the $2 \sigma$ limits from figure 6a of ref. [68]. While this approach leads to a strong bound when the real process $B \rightarrow K V, V \rightarrow \chi_{0} \chi_{0}$ dominates, these limits can be significantly weakened when the virtual process dominates, since the branching ratio accounts for a broader spread in $s_{B}$ bins.

We therefore also include partially integrated limits from the BaBar semileptonictagging analysis [69], which combined the world-leading limit on $B^{+} \rightarrow K^{+} \nu \nu$ with detailed information about the signal efficiencies as function of the momentum of the $K^{+}$(and hence on the missing energy). We select the $q^{2}$ ranges $\left[3.4^{2}, 4^{2}\right] \mathrm{GeV}^{2}$ and $\left[0,2.4^{2}\right] \mathrm{GeV}^{2}$ (corresponding to $p_{K}$ in the range $[1,1.5] \mathrm{GeV}$ and $\gtrsim 2 \mathrm{GeV}$, respectively), where the Boosted Decision Tree (BDT) efficiencies presented in figure 3 of ref. [69] are larger than $\sim 0.3$. This ensures that the signal efficiencies for our NP kinematics are of the same order of magnitude or higher than the ones for the SM signal. We then compare both regions with the low- $q^{2}$ and high- $q^{2} 95 \%$ C.L. limits, $\mathcal{B R}(B \rightarrow K+$ inv. $)<1.1 \times 10^{-5}$ and $\mathcal{B R}(B \rightarrow K+$ inv. $)<4.6 \times 10^{-5}$, respectively.

Open Access. This article is distributed under the terms of the Creative Commons Attribution License (CC-BY 4.0), which permits any use, distribution and reproduction in any medium, provided the original author(s) and source are credited.

\section{References}

[1] LHCb collaboration, Test of lepton universality using $B^{+} \rightarrow K^{+} \ell^{+} \ell^{-}$decays, Phys. Rev. Lett. 113 (2014) 151601 [arXiv: 1406.6482] [INSPIRE]. 
[2] LHCb collaboration, Test of lepton universality with $B^{0} \rightarrow K^{* 0} \ell^{+} \ell^{-}$decays, JHEP 08 (2017) 055 [arXiv : 1705.05802] [INSPIRE].

[3] LHCb collaboration, Search for lepton-universality violation in $B^{+} \rightarrow K^{+} \ell^{+} \ell^{-}$decays, Phys. Rev. Lett. 122 (2019) 191801 [arXiv:1903.09252] [INSPIRE].

[4] BeLLe collaboration, Test of lepton flavor universality in $B \rightarrow K^{*} \ell^{+} \ell^{-}$decays at Belle, arXiv: 1904.02440 [INSPIRE].

[5] LHCb collaboration, Angular analysis of the $B^{0} \rightarrow K^{* 0} \mu^{+} \mu^{-}$decay using $3 \mathrm{fb}^{-1}$ of integrated luminosity, JHEP 02 (2016) 104 [arXiv:1512.04442] [INSPIRE].

[6] BeLLE collaboration, Lepton-flavor-dependent angular analysis of $B \rightarrow K^{*} \ell^{+} \ell^{-}$, Phys. Rev. Lett. 118 (2017) 111801 [arXiv:1612.05014] [INSPIRE].

[7] CMS collaboration, Measurement of angular parameters from the decay $\mathrm{B}^{0} \rightarrow \mathrm{K}^{* 0} \mu^{+} \mu^{-}$in proton-proton collisions at $\sqrt{s}=8 \mathrm{TeV}$, Phys. Lett. B $\mathbf{7 8 1}$ (2018) 517 [arXiv:1710.02846] [INSPIRE].

[8] ATLAS collaboration, Angular analysis of $B_{d}^{0} \rightarrow K^{*} \mu^{+} \mu^{-}$decays in pp collisions at $\sqrt{s}=8$ TeV with the ATLAS detector, JHEP 10 (2018) 047 [arXiv: 1805.04000] [INSPIRE].

[9] LHCb collaboration, Angular analysis and differential branching fraction of the decay $B_{s}^{0} \rightarrow \phi \mu^{+} \mu^{-}$, JHEP 09 (2015) 179 [arXiv:1506.08777] [INSPIRE].

[10] LHCb collaboration, Differential branching fractions and isospin asymmetries of $B \rightarrow K^{(*)} \mu^{+} \mu^{-}$decays, JHEP 06 (2014) 133 [arXiv: 1403.8044] [INSPIRE].

[11] LHCb collaboration, Measurements of the $S$-wave fraction in $B^{0} \rightarrow K^{+} \pi^{-} \mu^{+} \mu^{-}$decays and the $B^{0} \rightarrow K^{*}(892)^{0} \mu^{+} \mu^{-}$differential branching fraction, JHEP 11 (2016) 047 [Erratum ibid. 04 (2017) 142] [arXiv: 1606.04731] [INSPIRE].

[12] W. Altmannshofer and D.M. Straub, New physics in $b \rightarrow s$ transitions after LHC run 1, Eur. Phys. J. C 75 (2015) 382 [arXiv:1411.3161] [InSPIRE].

[13] W. Altmannshofer, C. Niehoff, P. Stangl and D.M. Straub, Status of the $B \rightarrow K^{*} \mu^{+} \mu^{-}$ anomaly after Moriond 2017, Eur. Phys. J. C 77 (2017) 377 [arXiv:1703.09189] [InSPIRE].

[14] B. Capdevila, A. Crivellin, S. Descotes-Genon, J. Matias and J. Virto, Patterns of new physics in $b \rightarrow s \ell^{+} \ell^{-}$transitions in the light of recent data, JHEP 01 (2018) 093 [arXiv: 1704.05340] [INSPIRE].

[15] W. Altmannshofer, P. Stangl and D.M. Straub, Interpreting hints for lepton flavor universality violation, Phys. Rev. D 96 (2017) 055008 [arXiv: 1704.05435] [INSPIRE].

[16] G. D'Amico et al., Flavour anomalies after the $R_{K^{*}}$ measurement, JHEP 09 (2017) 010 [arXiv: 1704.05438] [INSPIRE].

[17] M. Ciuchini et al., On flavourful easter eggs for new physics hunger and lepton flavour universality violation, Eur. Phys. J. C 77 (2017) 688 [arXiv:1704.05447] [INSPIRE].

[18] A.K. Alok, B. Bhattacharya, A. Datta, D. Kumar, J. Kumar and D. London, New physics in $b \rightarrow s \mu^{+} \mu^{-}$after the measurement of $R_{K^{*}}$, Phys. Rev. D 96 (2017) 095009 [arXiv: 1704.07397] [INSPIRE].

[19] T. Hurth, F. Mahmoudi and S. Neshatpour, Global fits to $b \rightarrow$ sll data and signs for lepton non-universality, JHEP 12 (2014) 053 [arXiv:1410.4545] [INSPIRE].

[20] T. Hurth, F. Mahmoudi and S. Neshatpour, On the anomalies in the latest LHCb data, Nucl. Phys. B 909 (2016) 737 [arXiv:1603.00865] [InSPIRE]. 
[21] V.G. Chobanova, T. Hurth, F. Mahmoudi, D. Martinez Santos and S. Neshatpour, Large hadronic power corrections or new physics in the rare decay $B \rightarrow K^{*} \mu^{+} \mu^{-}$?, JHEP 07 (2017) 025 [arXiv: 1702.02234] [INSPIRE].

[22] T. Hurth, F. Mahmoudi, D. Martinez Santos and S. Neshatpour, Lepton nonuniversality in exclusive $b \rightarrow$ sll decays, Phys. Rev. D 96 (2017) 095034 [arXiv: 1705.06274] [INSPIRE].

[23] A. Arbey, T. Hurth, F. Mahmoudi and S. Neshatpour, Hadronic and new physics contributions to $b \rightarrow s$ transitions, Phys. Rev. D 98 (2018) 095027 [arXiv:1806.02791] [INSPIRE].

[24] M. Algueró et al., Emerging patterns of New Physics with and without Lepton Flavour Universal contributions, Eur. Phys. J. C 79 (2019) 714 [Addendum ibid. 80 (2020) 511] [arXiv: 1903.09578] [INSPIRE].

[25] A.K. Alok, A. Dighe, S. Gangal and D. Kumar, Continuing search for new physics in $b \rightarrow s \mu \mu$ decays: two operators at a time, JHEP 06 (2019) 089 [arXiv:1903.09617] [INSPIRE].

[26] M. Ciuchini et al., New physics in $b \rightarrow s \ell^{+} \ell^{-}$confronts new data on lepton universality, Eur. Phys. J. C 79 (2019) 719 [arXiv:1903.09632] [INSPIRE].

[27] A. Datta, J. Kumar and D. London, The $B$ anomalies and new physics in $b \rightarrow s e^{+} e^{-}$, Phys. Lett. B 797 (2019) 134858 [arXiv:1903.10086] [INSPIRE].

[28] J. Aebischer, W. Altmannshofer, D. Guadagnoli, M. Reboud, P. Stangl and D.M. Straub, B-decay discrepancies after Moriond 2019, Eur. Phys. J. C 80 (2020) 252 [arXiv: 1903.10434] [INSPIRE].

[29] K. Kowalska, D. Kumar and E.M. Sessolo, Implications for new physics in $b \rightarrow$ s $\mu \mu$ transitions after recent measurements by Belle and LHCb, Eur. Phys. J. C 79 (2019) 840 [arXiv: 1903.10932] [INSPIRE].

[30] A. Arbey, T. Hurth, F. Mahmoudi, D.M. Santos and S. Neshatpour, Update on the $b \rightarrow s$ anomalies, Phys. Rev. D 100 (2019) 015045 [arXiv:1904.08399] [INSPIRE].

[31] S. Bhattacharya, A. Biswas, S. Nandi and S.K. Patra, Exhaustive model selection in $b \rightarrow \mathrm{sl \ell}$ decays: pitting cross-validation against the Akaike information criterion, Phys. Rev. D 101 (2020) 055025 [arXiv: 1908.04835] [INSPIRE].

[32] A. Datta, J. Liao and D. Marfatia, A light $Z^{\prime}$ for the $R_{K}$ puzzle and nonstandard neutrino interactions, Phys. Lett. B $\mathbf{7 6 8}$ (2017) 265 [arXiv:1702.01099] [INSPIRE].

[33] F. Sala and D.M. Straub, A new light particle in B decays?, Phys. Lett. B 774 (2017) 205 [arXiv: 1704.06188] [INSPIRE].

[34] A. Datta, J. Kumar, J. Liao and D. Marfatia, New light mediators for the $R_{K}$ and $R_{K^{*}}$ puzzles, Phys. Rev. D 97 (2018) 115038 [arXiv:1705.08423] [InSPIRE].

[35] W. Altmannshofer et al., Light resonances and the low- $q^{2}$ bin of $R_{K^{*}}, J H E P 03$ (2018) 188 [arXiv: 1711.07494] [INSPIRE].

[36] A. Datta, J.L. Feng, S. Kamali and J. Kumar, Resolving the $(g-2)_{\mu}$ and B anomalies with leptoquarks and a dark Higgs boson, Phys. Rev. D 101 (2020) 035010 [arXiv:1908.08625] [INSPIRE].

[37] J. Lyon and R. Zwicky, Resonances gone topsy turvy - The charm of QCD or new physics in $b \rightarrow s \ell^{+} \ell^{-}$?, arXiv:1406.0566 [INSPIRE].

[38] LHCb collaboration, Angular analysis of the $B^{0} \rightarrow K^{* 0} e^{+} e^{-}$decay in the low- $q^{2}$ region, JHEP 04 (2015) 064 [arXiv: 1501.03038] [INSPIRE]. 
[39] B. Gripaios, M. Nardecchia and S.A. Renner, Linear flavour violation and anomalies in $B$ physics, JHEP 06 (2016) 083 [arXiv: 1509.05020] [INSPIRE].

[40] P. Arnan, L. Hofer, F. Mescia and A. Crivellin, Loop effects of heavy new scalars and fermions in $b \rightarrow s \mu^{+} \mu^{-}$, JHEP 04 (2017) 043 [arXiv: 1608.07832] [INSPIRE].

[41] J.M. Cline and J.M. Cornell, $R\left(K^{(*)}\right)$ from dark matter exchange, Phys. Lett. B 782 (2018) 232 [arXiv: 1711.10770] [inSPIRE].

[42] A. Crivellin, C. Greub, D. Müller and F. Saturnino, Importance of loop effects in explaining the accumulated evidence for new physics in B decays with a vector leptoquark, Phys. Rev. Lett. 122 (2019) 011805 [arXiv: 1807.02068] [INSPIRE].

[43] A. Datta, B. Dutta, S. Liao, D. Marfatia and L.E. Strigari, Neutrino scattering and B anomalies from hidden sector portals, JHEP 01 (2019) 091 [arXiv:1808.02611] [INSPIRE].

[44] B. Barman, D. Borah, L. Mukherjee and S. Nandi, Correlating the anomalous results in $b \rightarrow s$ decays with inert Higgs doublet dark matter and muon $(g-2)$, Phys. Rev. D 100 (2019) 115010 [arXiv:1808.06639] [INSPIRE].

[45] C. Marzo, L. Marzola and M. Raidal, Common explanation to the $R_{K^{(*)}}, R_{D^{(*)}}$ and $\epsilon^{\prime} / \epsilon$ anomalies in a $3 H D M+\nu_{R}$ and connections to neutrino physics, Phys. Rev. D 100 (2019) 055031 [arXiv: 1901.08290] [INSPIRE].

[46] P. Arnan, A. Crivellin, M. Fedele and F. Mescia, Generic loop effects of new scalars and fermions in $b \rightarrow s \ell^{+} \ell^{-}$and a vector-like $4^{\text {th }}$ generation, JHEP 06 (2019) 118 [arXiv: 1904.05890] [INSPIRE].

[47] J. Kawamura, S. Okawa and Y. Omura, Interplay between the $b \rightarrow$ sll anomalies and dark matter physics, Phys. Rev. D 96 (2017) 075041 [arXiv:1706. 04344] [INSPIRE].

[48] ATLAS collaboration, Search for supersymmetry in events with b-tagged jets and missing transverse momentum in pp collisions at $\sqrt{s}=13 \mathrm{TeV}$ with the ATLAS detector, JHEP 11 (2017) 195 [arXiv: 1708.09266] [INSPIRE].

[49] ATLAS collaboration, Search for a scalar partner of the top quark in the jets plus missing transverse momentum final state at $\sqrt{s}=13 \mathrm{TeV}$ with the ATLAS detector, JHEP 12 (2017) 085 [arXiv: 1709.04183] [INSPIRE].

[50] CMS collaboration, Search for direct top squark pair production in events with one lepton, jets, and missing transverse momentum at $13 \mathrm{TeV}$ with the CMS experiment, JHEP 05 (2020) 032 [arXiv: 1912.08887] [INSPIRE].

[51] CMS collaboration, Search for supersymmetry in proton-proton collisions at $13 \mathrm{TeV}$ in final states with jets and missing transverse momentum, JHEP 10 (2019) 244 [arXiv: 1908.04722] [INSPIRE].

[52] L. Di Luzio, M. Kirk and A. Lenz, Updated $B_{s}$-mixing constraints on new physics models for $b \rightarrow s \ell^{+} \ell^{-}$anomalies, Phys. Rev. D 97 (2018) 095035 [arXiv: 1712.06572] [INSPIRE].

[53] K. Kowalska and E.M. Sessolo, Expectations for the muon $g-2$ in simplified models with dark matter, JHEP 09 (2017) 112 [arXiv:1707.00753] [INSPIRE].

[54] CMS collaboration, Searches for pair production of charginos and top squarks in final states with two oppositely charged leptons in proton-proton collisions at $\sqrt{s}=13 \mathrm{TeV}, \mathrm{JHEP} 11$ (2018) 079 [arXiv: 1807.07799] [INSPIRE].

[55] ATLAS collaboration, Search for electroweak production of charginos and sleptons decaying into final states with two leptons and missing transverse momentum in $\sqrt{s}=13 \mathrm{TeV} p p$ collisions using the ATLAS detector, Eur. Phys. J. C 80 (2020) 123 [arXiv:1908.08215] [INSPIRE]. 
[56] Muon G-2 collaboration, Final report of the muon E821 anomalous magnetic moment measurement at BNL, Phys. Rev. D 73 (2006) 072003 [hep-ex/0602035] [INSPIRE].

[57] M. Davier, Update of the hadronic vacuum polarisation contribution to the muon $g-2$, Nucl. Part. Phys. Proc. 287-288 (2017) 70 [arXiv:1612.02743] [INSPIRE].

[58] F. Jegerlehner, Muon g-2 theory: the hadronic part, EPJ Web Conf. 166 (2018) 00022 [arXiv: 1705.00263] [INSPIRE].

[59] W. Altmannshofer, S. Gori, M. Pospelov and I. Yavin, Neutrino trident production: a powerful probe of new physics with neutrino beams, Phys. Rev. Lett. 113 (2014) 091801 [arXiv: 1406.2332] [INSPIRE].

[60] CCFR collaboration, Neutrino tridents and WZ interference, Phys. Rev. Lett. 66 (1991) 3117 [INSPIRE].

[61] CHARM-II collaboration, First observation of neutrino trident production, Phys. Lett. B 245 (1990) 271 [INSPIRE].

[62] P. Ko, Y. Omura and C. Yu, Higgs phenomenology in Type-I 2HDM with $\mathrm{U}(1)_{H}$ Higgs gauge symmetry, JHEP 01 (2014) 016 [arXiv:1309.7156] [INSPIRE].

[63] J.A. Gracey, Three loop MS-bar tensor current anomalous dimension in QCD, Phys. Lett. B 488 (2000) 175 [hep-ph/0007171] [INSPIRE].

[64] CMS collaboration, Measurement of the $B_{s}^{0} \rightarrow \mu^{+} \mu^{-}$branching fraction and search for $B^{0} \rightarrow \mu^{+} \mu^{-}$with the CMS experiment, Phys. Rev. Lett. 111 (2013) 101804 [arXiv: 1307.5025] [INSPIRE].

[65] CMS, LHCB collaboration, Observation of the rare $B_{s}^{0} \rightarrow \mu^{+} \mu^{-}$decay from the combined analysis of CMS and LHCb data, Nature $\mathbf{5 2 2}$ (2015) 68 [arXiv:1411.4413] [INSPIRE].

[66] LHCb collaboration, Measurement of the $B_{s}^{0} \rightarrow \mu^{+} \mu^{-}$branching fraction and effective lifetime and search for $B^{0} \rightarrow \mu^{+} \mu^{-}$decays, Phys. Rev. Lett. 118 (2017) 191801 [arXiv: 1703.05747] [INSPIRE].

[67] ATLAS collaboration, Study of the rare decays of $B_{s}^{0}$ and $B^{0}$ mesons into muon pairs using data collected during 2015 and 2016 with the ATLAS detector, JHEP 04 (2019) 098 [arXiv: 1812.03017] [INSPIRE].

[68] BABAR collaboration, Search for $B \rightarrow K^{(*)} \nu \bar{\nu}$ and invisible quarkonium decays, Phys. Rev. $D 87$ (2013) 112005 [arXiv: 1303.7465] [INSPIRE].

[69] BaBAR collaboration, Search for the rare decay $B \rightarrow K \nu \bar{\nu}$, Phys. Rev. D 82 (2010) 112002 [arXiv: 1009.1529] [INSPIRE].

[70] BeLLe collaboration, Search for $B \rightarrow h^{(*)} \nu \bar{\nu}$ with the full Belle $\Upsilon(4 S)$ data sample, Phys. Rev. D 87 (2013) 111103 [arXiv:1303.3719] [InSPIRE].

[71] Belle collaboration, Search for $\boldsymbol{B} \rightarrow \boldsymbol{h} \boldsymbol{\nu} \overline{\boldsymbol{\nu}}$ decays with semileptonic tagging at Belle, Phys. Rev. D 96 (2017) 091101 [Addendum ibid. 97 (2018) 099902] [arXiv:1702.03224] [INSPIRE].

[72] LHCb collaboration, Search for hidden-sector bosons in $B^{0} \rightarrow K^{* 0} \mu^{+} \mu^{-}$decays, Phys. Rev. Lett. 115 (2015) 161802 [arXiv: 1508.04094] [INSPIRE].

[73] T.R. Slatyer, Indirect dark matter signatures in the cosmic dark ages. I. Generalizing the bound on s-wave dark matter annihilation from Planck results, Phys. Rev. D 93 (2016) 023527 [arXiv: 1506.03811] [INSPIRE].

[74] J.A. Bailey et al., $B \rightarrow K l^{+} l^{-}$decay form factors from three-flavor lattice QCD, Phys. Rev. D 93 (2016) 025026 [arXiv: 1509.06235] [INSPIRE]. 
[75] F. Jegerlehner and A. Nyffeler, The muon g-2, Phys. Rept. 477 (2009) 1 [arXiv:0902.3360] [INSPIRE].

[76] F.S. Queiroz and W. Shepherd, New physics contributions to the muon anomalous magnetic moment: a numerical code, Phys. Rev. D 89 (2014) 095024 [arXiv:1403.2309] [InSPIRE].

[77] W. Altmannshofer, S. Gori, M. Pospelov and I. Yavin, Quark flavor transitions in $L_{\mu}-L_{\tau}$ models, Phys. Rev. D 89 (2014) 095033 [arXiv: 1403.1269] [InSPIRE].

[78] AlePh, Delphi, L3, OPAL, SLD, LeP Electroweak Working Group, SLD Electroweak Group, SLD Heavy Flavour Group collaboration, Precision electroweak measurements on the $Z$ resonance, Phys. Rept. 427 (2006) 257 [hep-ex/0509008] [INSPIRE].

[79] F. Bishara, U. Haisch and P.F. Monni, Regarding light resonance interpretations of the $B$ decay anomalies, Phys. Rev. D 96 (2017) 055002 [arXiv:1705.03465] [INSPIRE].

[80] BeLLE II collaboration, Search for an invisibly decaying $Z^{\prime}$ boson at Belle II in $e^{+} e^{-} \rightarrow \mu^{+} \mu^{-}\left(e^{ \pm} \mu^{\mp}\right)$ plus missing energy final states, Phys. Rev. Lett. 124 (2020) 141801 [arXiv: 1912.11276] [INSPIRE].

[81] L. Darmé, S.A.R. Ellis and T. You, Light dark sectors through the fermion portal, JHEP 07 (2020) 053 [arXiv: 2001.01490] [INSPIRE].

[82] J. Beacham et al., Physics beyond colliders at CERN: beyond the standard model working group report, J. Phys. G 47 (2020) 010501 [arXiv: 1901.09966] [InSPIRE].

[83] G. Durieux, F. Maltoni and C. Zhang, Global approach to top-quark flavor-changing interactions, Phys. Rev. D 91 (2015) 074017 [arXiv:1412.7166] [INSPIRE].

[84] M. Chala, J. Santiago and M. Spannowsky, Constraining four-fermion operators using rare top decays, JHEP 04 (2019) 014 [arXiv: 1809.09624] [INSPIRE].

[85] J. De Blas et al., HEPfit: a code for the combination of indirect and direct constraints on high energy physics models, Eur. Phys. J. C 80 (2020) 456 [arXiv:1910.14012] [InSPIRE].

[86] A. Caldwell, D. Kollar and K. Kroninger, BAT: the Bayesian Analysis Toolkit, Comput. Phys. Commun. 180 (2009) 2197 [arXiv: 0808. 2552] [INSPIRE].

[87] CLEO collaboration, Search for the familon via $B^{ \pm} \rightarrow \pi^{ \pm} X^{0}, B^{ \pm} \rightarrow K^{ \pm} X^{0}$, and $B^{0} \rightarrow K_{S}^{0} X^{0}$ decays, Phys. Rev. Lett. 87 (2001) 271801 [hep-ex/0106038] [INSPIRE].

[88] K. Griest and D. Seckel, Three exceptions in the calculation of relic abundances, Phys. Rev. D 43 (1991) 3191 [INSPIRE].

[89] R.T. D'Agnolo and J.T. Ruderman, Light dark matter from forbidden channels, Phys. Rev. Lett. 115 (2015) 061301 [arXiv: 1505.07107] [INSPIRE]. 